Pacific Journal of Mathematics

CENTRALIZERS OF TWISTED GROUP ALGEBRAS 


\section{CENTRALIZERS OF TWISTED GROUP ALGEBRAS}

\section{ROBERT C. BUSBY}

Centralizers (left, right, and double) of rings and algebras have received much attention recently, and seem likely to become an important topic in ring theory. They have proved quite useful in Banach algebra theory, and a good deal of work has been done on the computation of centralizers for various Banach algebras. In this paper we compute the left centralizers of a twisted group algebra, a generalization of the group algebra of locally compact group, which includes as special cases the covariance algebras of quantum field theory, and the group algebras of separable group extensions (explicitly given in terms of the subgroup algebra and quotient group). We give a representation of the algebra of left centralizers of a "locally continuous" twisted group algebra as an algebra of vector-valued measures with "twisted convolution'. This result gives more than explicit computation of centralizers. The form of the result enables us to investigate isometric isomorphisms between twisted group algebras along lines previously pursued for ordinary group algebras. In some cases we get a complete description of possible isomorphism classes in terms of orbits in a cohomology set.

Double Centralizers were first introduced by G. Hochschild in his cohomology studies [15], and later, independently, by B. E. Johnson in [16] (see also [17]), who was largely interested in applications to analysis. Work on the ideal theory of algebras and Banach algebras by Dauns and Hofmann ([6], [7]), and on extensions of $C^{*}$-algebras [1] are examples of such applications. Left centralizers are used in the papers of Wendel on norm decreasing isomorphisms of group algebras ([21], [22]). The latter results are especially important and suggestive for us. Wendel showed in [21] Th. 2 that the left centralizers of a group algebra form an algebra isometrically isomorphic with the algebra $M(G)$ of complex valued, bounded, regular Borel measures on $G$. This suggested the result given above, namely that centralizers of a twisted group algebra should be vector-valued measures. We also show, in analogy to a result of Wendel, that the isometric left centralizers correspond to certain measures with a one point support. Moreover, several of our arguments take those of Wendel as a starting point. The description of isometric isomorphisms of group algebras given in [21] is the forerunner of our results on classifying isometric isomorphisms of twisted group algebras, and, as a matter of fact, is a corollary of them. The form of our results on 
left centralizers opens up the possibility of extending Greenleaf's work ([13]) on norm decreasing homomorphisms of group algebras to the twisted group algebra setting (although we do not pursue the possibility here).

In $\S 1$, we collect the needed facts about centralizers. In $\S 2$ we give a general cohomology and extension discussion which is needed later. Section 3 is denoted to developing and expounding the theory of Banach algebra valued measures as we will need it. These first three sections contain summaries of some well known results, reorganization and extension of some lesser known material, and some new results. Section 4 contains the main description of left centralizers on a locally continuous twisted group algebra, as well as a discussion of double centralizers and isometric centralizers, and $\$ 5$ gives applications to the study of isomorphisms and particular examples. It should be noted that in the case where the object algebra is the complex numbers, the automorphism is trivial and the cocycle is continuous, some of the results of $\S 5$ specialize to results of Edwards and Lewis [11].

1. Centralizers on algebras. The results of this section will be stated without proofs, except when proofs do not exist elsewhere. In this entire paper, topological group will always mean second countable group, and the term Banach algebra will always include the assertions that the algebra is separable and has a norm one, two-sided, approximate identity. A Banach *-algebra will be a Banach algebra with an isometric involution. Isomorphism and representation of a Banach algebra (group) will always mean (unless otherwise stated) isometric (topological) isomorphism and continuous (continuous unitary) representation on a separable Hilbert space, respectively. If the algebras have involution, isomorphism and non-degenerate will also mean $*$-isomorphism and *-representation, respectively.

Let $A$ be a Banach algebra. A left (right) centralizer on $A$ is a bounded linear map $L: A \rightarrow A(R: A \rightarrow A)$ such that if $a, b \in A$, then $L(a b)=L(a) b(R(a b)=a R(b))$. A double centralizer on $A$ is a pair of functions, $(L, R)$, from $A$ to $A$ such that if $a, b \in A, a L(b)=R(a) b$. Let $\mathscr{L}_{L}(A), \mathscr{L}_{R}(A), \mathscr{L}(A)$ be the set of left, right, and double centralizers on $A$, respectively.

Proposition 1.1. $\|R\|$.

(i ) If $(L, R) \in \mathscr{C l}(A)$ then $L \in \mathscr{l}_{L}(A)$ and $R \in \mathscr{M}_{R}(A)$, and $\|L\|=$

(ii) $\mathscr{L}_{L}(A)$ and $\mathscr{C}_{R}(A)$ are Banach algebras with composition as multiplication and operator norm.

(iii) If $A$ is a Banach *-algebra, then $\mathscr{C l}(A)$ is a Banach *-algebra with operations as follows: 


$$
\begin{aligned}
\left(L_{1}, R_{1}\right) \circ\left(L_{2}, R_{2}\right) & =\left(L_{1} L_{2}, R_{2} R_{1}\right) ; \\
(L, R)^{*} & =\left(R^{\prime}, L^{\prime}\right) \text { where } R^{\prime}(x)=R\left(x^{*}\right)^{*} \text { and } L^{\prime}(x)=L\left(x^{*}\right)^{*} ; \\
\|(L, R)\| & =\|L\| \equiv\|R\| .
\end{aligned}
$$

(iv) The map from $\mathscr{L}(A)$ to $\mathscr{L}_{L}(A)$ which takes $(L, R)$ to $L$ is an isometric monomorphism of $\mathscr{C}(A)$ into $\mathscr{K}_{L}(A)$.

Proof. All these facts may be easily deduced from statements in [16], and our assumptions on $A$.

There is a natural map $i_{A}$ of $A$ into $\mathscr{A}_{L}(A)$ given by: $i_{A}(x)=L_{x}$ where, for $y \in A, L_{x}(y)=x y$. If $R_{x}$ is similarly defined by $R_{x}(y)=$ $y x$, then the map $\bar{i}_{A}$ given by $\bar{i}_{A}(x)=\left(L_{x}, R_{x}\right)$ is a map into $\mathscr{K}(A)$.

Proposition 1.2. (i) $i_{A}$ and $\bar{i}_{A}$ are isometric algebra homomorphisms,

(ii) if $A$ is a *-algebra, $\bar{i}_{A}$ preserves involution,

(iii) $i_{A}(A)\left(r e s p . \bar{i}_{A}(A)\right)$ is a left (resp. two-sided) ideal in

$$
\mathscr{H}_{L}(A)(\operatorname{resp} . \mathscr{M}(A)) \text {. }
$$

(iv) If we regard $A \subseteq \mathscr{H}(A) \subseteq \mathscr{H}_{L}(A)$ by the above identifications, then $\mathscr{C}(A)$ is the idealizer of $A$ in

$$
\mathscr{M}_{L}(A) \text {, i.e., }\left\{x \in \mathscr{C}_{L}(A) \mid(x \cdot A \cup A \cdot x) \subset A\right\} \text {. }
$$

Proof. The first three assertions are elementary. (iv) follows from Proposition 1.1, (iv) and the fact that if $A \cdot x \subset A$ for some $x \in$ $\mathscr{A}_{L}(A)$, then left and right multiplication by $x$ provide a double centralizer pair of actions.

In the future we will identify $A$ with $i_{A}(A)$ and with $\bar{i}_{A}(A)$. If $a \in A$ and $m=(L, R) \in \mathscr{C}(A)$ then (with the above identication in force) $m a=L(a)$ and $a m=R(a)$.

DEFINITION 1.3. (i) The strong topology on $\mathscr{K}_{L}(A)$ is the locally convex topology generated by the seminorms $\left(\lambda_{a} ; a \in A\right)$ where for $m \in$ $\mathscr{L}_{L}(A), \lambda_{a}(m)=\|m a\|$.

(ii) The strict topology on $\mathscr{C}(A)$ is the locally convex topology generated by the seminorms $\left(\lambda_{a} ; a \in A\right)$ and $\left(\rho_{a} ; a \in A\right)$ where $\lambda_{a}$ is as above and $\rho_{a}(m)=\|a m\|$.

Proposition 1.4. (i) $A$ is strongly (strictly) dense in $\mathscr{M}_{L}(A)(\mathscr{L}(A))$ and $\mathscr{M}_{L}(A)(\mathscr{L}(A))$ is strongly (strictly) complete.

(ii) The unit ball in $\mathscr{M}_{L}(A)(\mathscr{L}(A))$ is strongly (strictly) metrizable and complete.

(iii) If $A$ has involution, the group $\mathscr{U}(A)$ of unitaries in

$$
\mathscr{C}(A)\left(u^{*}=u^{-1}=1\right)
$$


is a Polish group in the strict topology (i.e., separable, and metrizable by a complete metric).

Proof. A proof of (i) can be deduced from [1], Propositions 3.5, 3.6. Let $\Lambda$ be the set of seminorms $\left\{\left(\lambda_{a}\right) \cup\left(\rho_{a}\right)\right\}$, where a ranges over a countable dense set in $A$. Then the sets $\{m \in \mathscr{L}(A) \mid \lambda(m)<\varepsilon\}$, where $\varepsilon$ is rational and $\lambda \in \Lambda$ can easily be shown to form a base for the unit ball in $A$. A similar result holds in $\mathscr{C}_{L}(A)$, and so (ii) is true. Finally it is easily shown that the strict limit of unitaries is unitary in $\mathscr{C}(A)$, so $\mathscr{C}(A)$ is closed in $\mathscr{C}(A)$, and (iii) follows. In this paper, $\mathscr{C}(A)$ will always be assumed to have the strict topology.

Proposition 1.5. Let $B$ be a Banach algebra which contains $A$ (isometrically) as a norm closed left ideal. Suppose also:

(i) $\|b\|=\sup _{\substack{\| x_{i} \mid \leq 1 \\ x \in A}}\|b x\|$ for any $b \in B$ and

(ii) If $\left(b_{i}\right)_{i \triangleq I}$ is a net in $B$ such that $\lim _{i, j \rightarrow \infty}\left\|b_{i} a-b_{j} a\right\|=0 \forall a \epsilon$ $A$, and $\left\|b_{i}\right\| \leqq 1 \forall_{i}$, then $\exists b_{\infty} \in B$ such that $\lim _{i \rightarrow \infty} b_{i} a=b_{\infty} a \forall a \in A$. Then: (1) The function $\lambda_{b}$ from $A$ to $A$ given by $\lambda_{b}(a)=b a$ is in $\mathscr{L}_{L}(A), \forall b \in B$ and

(2) The map $A$, which takes $b$ to $\lambda_{b}$, is an isomorphism of $B$ onto $\mathscr{C l}_{L}(A)$.

Proof. (1) is trivial, as is the fact that $\Lambda$ is homomorphism. Condition (i) shows that $\Lambda$ is isometric. To show $\Lambda$ is onto, choose $m \in \mathscr{C}_{L}(A)$. By the linearity of $\Lambda$, we may assume $\|m\|=1$. Now it is not hard to show that if $\left(e_{i}\right)_{i \in I}$ is an approximate identity (always two-sided, norm-one) in $A$, and $b_{i}=m e_{i}$, then $\lim _{i \rightarrow \infty} b_{i}=m$ (strongly). Also $\left(b_{i}\right)_{i \in I}$ satisfies Condition (ii) above and so $\lim _{i \rightarrow \infty} b_{i} a=b a$ for some $b \in B$ and all $a \in A$. Then $m=\lambda_{b}$ and $A$ is onto.

\section{Proposition 1.6.}

(i) If $\phi$ is an isomorphism between Banach algebras (resp. Banach *-algebras) $A_{1}$ and $A_{2}$, then $\phi$ uniquely extends to an isomorphism (also denoted $\phi$ ) between $\mathscr{C}_{L}\left(A_{1}\right)$ and $\mathscr{M}_{L}\left(A_{2}\right)$ (resp. $\mathscr{C}\left(A_{1}\right)$ and $\mathscr{C}\left(A_{2}\right)$ ).

(ii) $\dot{\phi}$ induces a homeomorphism from $\mathscr{U}\left(A_{1}\right)$ onto $\mathscr{U}\left(A_{2}\right)$ (each with the strict topology).

(iii) If $\pi$ is a representation of the Banach (resp. Banach*) algebra $A$ on $H$, then $\pi$ extends uniquely to a representation of $\mathscr{C}_{L}(A)$ (resp. $\mathscr{l l}(A)$ ) on $H$. The extended representation is also denoted $\pi$.

Proof. (i) and (iii) can be found in [16], and (ii) is straight forward. 
Our final result on centralizers is a technical result which will be needed in $\S 4$. We first prove a lemma.

Lemma 1.7. Let $A$ be a Banach algebra and $m \in \mathscr{A}_{L}(A)$. Then if $\left(e_{i}\right)_{i \in I}$ is an approximate identity in $A$;

$$
\|m\|=\lim _{i \rightarrow \infty}\left\|m e_{i}\right\| \text {. }
$$

Proof.

$$
\begin{aligned}
\|m\| & =\sup _{\|a\|=1}\|m a\| \\
& =\sup _{\|a\|=1}\left[\lim _{i \rightarrow \infty}\left\|m e_{i} a\right\|\right] \leqq \lim _{i \rightarrow \infty}\left[\sup _{\|a\|=1}\left\|m e_{i} a\right\|\right] \\
& =\lim _{i \rightarrow \infty}\left\|m e_{i}\right\| \leqq \varlimsup_{i \rightarrow \infty}\left\|m e_{i}\right\| \leqq\|m\| .
\end{aligned}
$$

Corollary 1.8. If $m \in \mathscr{M}_{L}(A)$ and $\forall a \in A$,

$$
\|m a\|=\|m\|\|a\| \text {, then }\|m n\|=\|m\|\|n\| \forall n \in \mathscr{M}_{L}(A) \text {. }
$$

Proof. $\|m n\|=\lim _{i \rightarrow \infty}\left\|(m n) e_{i}\right\|\left(\left(e_{i}\right)_{i \in I}\right.$ an approximate identity in $A$ )

$$
\begin{aligned}
& =\lim _{i \rightarrow \infty}\left\|m\left(n e_{i}\right)\right\| \\
& =\|m\| \lim _{i \rightarrow \infty}\left\|n e_{i}\right\|=\|m\|\|n\| .
\end{aligned}
$$

Proposition 1.9. Suppose that $m, n \in \mathscr{M}_{L}(A)$ for some Banach algebra $A$, and for all $a, b \in A,\|m a+n b\|=\|m\|\|a\|+\|n\|\|b\|$. Then $n=0$ or $m=0$.

Proof. As in Corollary 1.8 we can show that if $r, s \in \mathscr{A}_{L}(A)$, then $\|m r+n s\|=\|m\|\|r\|+\|n\|\|s\|$. Thus the above norm equality holds in $\mathscr{C}_{L}(A)$. Let $n \neq 0$. If $c_{1}$ and $c_{2}$ are complex constants, we have (letting $r=c_{2} n$ and $s=c_{1}$ )

$$
\left\|c_{2} m n+c_{1} n\right\|=\left|c_{2}\right|\|m\|\|n\|+\left|c_{1}\right|\|n\|=\left(\left|c_{2}\right|\|m\|+\left|c_{1}\right|\right)\|n\|,
$$

which implies that

$$
\left\|c_{2} m+c_{1}\right\|=\left|c_{2}\right|\|m\|+\left|c_{1}\right|
$$

Similarly

$$
\begin{aligned}
& \left\|c_{3} m^{2} n+c_{2} m n+c_{1} n\right\|=\left\|m\left(c_{3} m n+c_{2} n\right)+c_{1} n\right\| \\
= & \|m\|\left\|c_{3} m n+c_{2} n\right\|+\left|c_{1}\right|\|n\|=\left|c_{3}\right|\|m\|^{2}\|n\| \\
& +\left|c_{2}\right|\|m\|\|n\|+\left|c_{1}\right|\|n\|,
\end{aligned}
$$

which implies that 


$$
\left\|c_{3} m^{2}+c_{2} m+c_{1}\right\|=\left|c_{3}\right|\|m\|^{2}+\left|c_{2}\right|\|m\|+\left|c_{1}\right| \text {. }
$$

By induction we can show that if $c_{1}, \cdots, c_{n}$ are complex constants then

$$
\left\|c_{n} m^{n-1}+\cdots+c_{2} m+c_{1}\right\|=\left|c_{n}\right|\|m\|^{n-1}+\cdots+\left|c_{2}\right|\|m\|+\left|c_{1}\right| .
$$

In particular if $p$ and $q$ are polynomials with complex coefficients then $\|p(m) \cdot q(m)\|=\|p(m)\|\|q(m)\|$. Let $B$ be the (commutative) Banach subalgebra of $\mathscr{L}_{L}(A)$ generated by $m$ and $I$. Since polynomials in $m$ are norm dense in $B,\left\|b_{1} b_{2}\right\|=\left\|b_{1}\right\|\left\|b_{2}\right\|$ for all $b_{1}, b_{2}$ in $B$. If $b \in$ $B$, the spectral radius of $b=\lim _{n \rightarrow \infty}\left\|b^{n}\right\|^{1 / n}=\|b\|$, and so if $X$ is the maximal ideal space of $B$, the Gelfand map $b \rightarrow \hat{b}$ of $B$ into $C(X)$ is isometric. Now for each $b \in B$, let $S(b)=\{x \in X|| \hat{b}(x) \mid=\|b\|\}$. Then $S(b)$ is a nonempty compact subset of $X$ for all $b$ in $B$. If there are elements $b_{1}, \cdots, b_{n}$ in $B$ such that $\bigcap_{i=1}^{n} S\left(b_{i}\right)=\phi$, then for any $x \in X \exists i$ with $\left|\hat{b}_{i}(x)\right|<\left\|b_{i}\right\|$. This means that $\left\|b_{i} \cdots b_{n}\right\|=\max _{x \in X} \mid \hat{b}_{1}(x) \cdots$ $\hat{b}_{n}(x) \mid<\left\|b_{i}\right\| \cdots\left\|b_{n}\right\|$, which is a contradiction. The collection $(S(b))_{b \in B}$ thus has the finite intersection property and so $\bigcap_{b \in B} S(b)$ contains a point $x_{0}$. We must have $\|b\|=\left|\hat{b}\left(x_{0}\right)\right| \forall b \in B$. If $m \neq 0$ and $c=\hat{m}\left(x_{0}\right) \neq$ 0 , let $b=c^{-1} m$. Then $\hat{b}\left(x_{0}\right)=1$ and $\widehat{b}^{2}\left(x_{0}\right)=\left(\hat{b}\left(x_{0}\right)\right)^{2}=1$, so $\left(\widehat{b^{2}-b}\right)\left(x_{0}\right)=$ 0 and $\left\|b^{2}-b\right\|=0$. We finally see that $0=\left\|b^{2}-b\right\|=\| c^{-2} m^{2}-$ $c^{-1} m\left\|=|c|^{-2}\right\| m\left\|^{2}+|c|^{-1}\right\| m \|$ so $\|m\|=0$. This contradiction proves that $m=0$.

2. Cohomology considerations. Throughout this section, $G$ will be a fixed topological group.

Definition 2.1. (a) Let $A$ be a topological group (resp. Banach *-algebra) and $\mathscr{A}(A)$ the group of automorphisms of $A$. $\mathscr{A}(A)$ will always have the pointwise convergence topology. A cohomology pair (also called twisting pair) for the pair $(G, A)$ is a pair $(T, \alpha)$ where $T: G \rightarrow$ $\mathscr{A}(A)$ and $\alpha: G \times G \rightarrow A$ (resp. $\mathscr{C}(A))$ are such that:

(i) $T$ and $\alpha$ are Borel measurable,

(ii) $T(x) \alpha(y, z) \alpha(x, y z)=\alpha(x, y) \alpha(x y, z) \equiv \beta(x, y, z)$ for all $x, y, z$ in $G(T(x)$ extended to $\mathscr{C}(A)$ is used here if $A$ is a Banach $*$-algebra),

(iii) $[T(x) T(y) a] \alpha(x, y)=\alpha(x, y)(T(x y) \alpha)$ for all $x, y$ in $G, a \in A$.

(iv) $\alpha(x, e)=\alpha(e, y)=I$ for $x, y$ in $G$ where $e$ and $I$ are respective identities in $G$ and $A$ (resp. $\mathscr{U}(A)$ ).

(b) We say that $(T, \alpha)$ is a locally continuous pair if (i) $T$ is continuous in a neighborhood $N$ of $e$, and the map $(x, a) \rightarrow T(x) a$ is continuous from $N \times A \rightarrow A$. Also $\alpha$ is continuous on $N \times N$,

(ii) For each $x$ in $G$ there is a neighborhood $N_{x}$ of $e$ such that $\beta\left(x^{-1}, y, x\right)$ is continuous in $y$ for $y$ in $N_{x}$.

We remark that we could have given an analogous definition of continuous at the identity (where $N$ and all $N_{x}$ are replaced by $\{e\}$ ), 
and that this and the above definitions are essentially those of Calabi [5].

Also notice that if $A$ is a Banach *-algebra and $(T, \alpha)$ is a cohomology pair for $(G, A)$, then $(T, \alpha)$ is also a cohomology pair for $(G, \mathscr{U}(A)$ ) (recall that $T$ extends to $\mathscr{C}(A) \supset A)$. The only non obvious part of this assertion is contained in the following lemma.

Lemma 2.2. If $A$ is a Banach *-algebra, $(T, \alpha)$ is a cohomology pair for $(G, A)$, and $m \in \mathscr{C}(A)$, then the function $f(x)=T(x) m$ is Borel from $G$ to $\mathscr{C}(A)$, the latter with the strict topology.

Proof. Let $a \in A$. Then the function $x \rightarrow T(x)^{-1} a$ is norm bounded and Borel, and since $A$ is separable, this function is the uniform limit of functions $\sum_{n=1}^{\infty} \chi_{B_{n}} \cdot a_{n}$, where the $B_{n}$ are disjoint Borel sets in $G, a_{n} \in A$, and $\chi_{B_{n}}$ is the characteristic function of $B_{n}$. Now $x \rightarrow T(x)\left(\sum_{n=1}^{\infty} \chi_{B_{n}}(m \cdot a)\right)$ is clearly Borel from $G$ to $A$ and thus so is the function

$$
x \rightarrow T(x)\left(m\left(T(x)^{-1} a\right)\right)=(T(x) m) \alpha .
$$

This completes the proof.

It can be similarly proved that if $(T, \alpha)$ is locally continuous for $(G, A)$, then (extended) it is also locally continuous for $(G, \mathscr{C}(A))$.

Again let $A$ be a topological group (Banach *-algebra). We let $Z^{2}(G, A)$ be the set of all locally continuous cohomology pairs for $(G, A)$. Let $F(G, A)$ be the set of all functions from $G$ to $A$ (resp. $\mathscr{U}(A)$ ) which are Borel measurable and continuous in a neighborhood of the identity. Then $F(G, A)$ is a group under pointwise multiplication and acts on $Z^{2}(G, A)$ as follows:

$$
\text { If } \tau=(T, \alpha) \in Z^{2}(G, A) \text { and } p \in F(G, A)
$$

then

$$
p \cdot \tau=\left(T_{p}, \alpha_{p}\right) \in Z^{2}(G, A)
$$

where

$$
T_{p}(x) a=p(x)(T(x) a) p(x)^{-1}
$$

and

$$
\alpha_{p}(x, y)=p(x)(T(x) p(y)) \alpha(x, y) p(x y)^{-1}
$$

for all $x, y$ in $G$ and $a$ in $A$.

LEMma 2.3. The above action defines $F(G, A)$ as a transformation group of $Z^{2}(G, A)$. 
Proof. It is not hard to show that if $p, q \in F(G, A), \tau \in Z^{2}(G, A)$, then $p \cdot(q \cdot \tau)=(p q) \cdot \tau$. The fact that $p \cdot \tau$ is in $Z^{2}(G, A)$ follows from the continuity of $p$ in a neighborhood of $e$, and straightforward calculations.

The resulting orbit space $Z^{2}(G, A) / F(G, A)$ is called the 2-cohomology set of $(G, A)$, and denoted $H^{2}(G, A)$.

Let $C(A)$ be the center of $A(\operatorname{resp} . \mathscr{C}(A))$. If $(T, \alpha) \equiv \tau \in Z^{2}(G, A)$ is given, then it is not hard to show that the stability subgroup $F_{\tau}$ of $F(G, A)$ at $\tau$ is the set of functions $p$ in $F(G, A)$ such that $p$ has values in $C(A)$, and $p(x)(T(x) p(y)) p(x y)^{-1}=I . \quad F_{\tau}$ is an abelian group denoted $Z^{1}(G, C(A))$, the Borel, locally continuous 1-cocycles of $G$ with respect to $T$, having values in $C(A)$.

We remark that if $A$ is abelian then $Z^{2}(G, A)$ is the disjoint union of the usual cocycle groups $Z_{T}^{2}(G, A)$, and $H^{2}(G, A)$ is just the disjoint union of the cohomology groups $H_{T}^{2}(G, A)$, where $T$ ranges over the continuous homomorphisms of $G$ into $\mathscr{A}(A)$, and all cochains are Borel and locally continuous.

Now let $G_{1}$ and $G_{2}$ be locally compact groups and $A_{1}, A_{2}$ topological groups (Banach *-algebras). Let $\phi: G_{1} \rightarrow G_{2}$ and $\gamma: A_{1} \rightarrow A_{2}$ be isomorphisms, and $\tau=(T, \alpha) \in Z^{2}\left(G_{2}, A_{2}\right)$. Let $s=(\dot{\phi}, \gamma)$ and define the pair $s \cdot \tau \equiv\left(T_{s}, \alpha_{s}\right)$ by:

$$
\left.T_{s}(x)(\alpha)=\gamma^{-1}[T(\dot{\phi}(x))(\gamma \alpha))\right]
$$

and

$\alpha_{s}(x, y)=\gamma^{-1}(\alpha(\phi(x), \phi(y))) \quad$ (where if $A$ is a Banach $*$-algebra, $\gamma^{-1}$ is canonically extended to $\left.\mathscr{C}_{L}\left(A_{2}\right)\right)$.

LEMma 2.4. (i) $s \cdot \tau \in Z^{2}\left(G_{1}, A_{1}\right)$

(ii) if $\tau_{1}$ and $\tau_{2} \in Z^{2}\left(G_{2}, A_{2}\right)$ and $\left[\tau_{1}\right]=\left[\tau_{2}\right]$ in $H^{2}\left(G_{2}, A_{2}\right)$ ([r] represents the equivalence class of $\tau$ ) then

$$
\left[s \cdot \tau_{1}\right]=\left[s \cdot \tau_{2}\right] \text { in } H^{2}\left(G_{1}, A_{1}\right) \text {. }
$$

Proof. The computations involved in (i) are straightforward, as are the measurability and continuity properties. If $s=(\phi, \gamma)$ in (ii) and $\tau_{2}=p \cdot \tau_{1}$ for $p \in F\left(G_{2}, A_{2}\right)$, then it is easily shown that $q \cdot\left(s \cdot \tau_{1}\right)=$ $\left(s \cdot \tau_{2}\right)$ where $q \in F\left(G_{1}, A_{1}\right)$ is given by: $q(x)=\gamma^{-1} p(\phi(x))$.

Corollary 2.5. $\mathscr{G}(G, A) \equiv \mathscr{A}(G) \times \mathscr{A}(A)$ acts as a transformation group on $H^{2}(G, A)$ with action as given above.

Now let $A$ be a (not necessarily topological) group and $\tau \equiv(T, \alpha)$ 
be a pair satisfying (ii), (iii), and (iv) of Definition 2.1 a. We then call $\tau$ an algebraic cohomology pair for $(G, A)$. Let $B_{\tau}$ be the group $A \times G$ with multiplication $\left(a_{1}, g_{1}\right) \circ\left(a_{2}, g_{2}\right)=\left(a_{1} T\left(g_{1}\right)\left(a_{2}\right) \alpha\left(g_{1}, g_{2}\right), g_{1} g_{2}\right)$. Let $i_{\tau}(a)=(a, 0)$ and $\pi_{\tau}(a, g)=g$ define maps from $A$ to $B_{\tau}$ and $B_{\tau}$ to $G$ respectively. Then, as is well known, $0 \rightarrow A \stackrel{i_{\tau}}{\longrightarrow} B_{\tau} \stackrel{\pi_{\tau}}{\longrightarrow} G \rightarrow 0$ is an exact sequence of groups, which we denote by $E_{\tau}$. We say that two exact sequences $E_{k}: 0 \longrightarrow A \stackrel{i_{k}}{\longrightarrow} B_{k} \stackrel{\pi_{k}}{\longrightarrow} G \longrightarrow 0(k=1,2)$ are isomorphic if there are maps $\gamma: A \rightarrow A, \beta: B_{1} \rightarrow B_{2}, \phi: G \rightarrow G$, all algebraic isomorphisms, making the following diagram commutative:

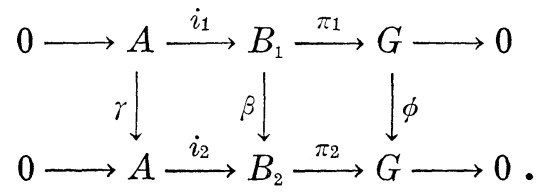

The triple $(\gamma, \beta, \phi)$ is called an isomorphism of $E_{1}$ with $E_{2}$. If $\gamma$ and $\phi$ are each the respective identity maps on $A$ and $G$, we say that $E_{1}$ and $E_{2}$ are equivalent, and write $E_{1} \cong E_{2}$. For a given exact sequence $E$, we let $[E]$ denote the equivalence class containing $E$, and we denote the set of equivalence classes of such exact sequences (for fixed $A$ and $G)$ by $\operatorname{Ext}(G, A)$. It is well known that the correspondence $\tau \rightarrow\left[E_{\tau}\right]$ is a bijection from the set of algebraic cohomology pairs for $(G, A)$ to $\operatorname{Ext}(G, A)$.

Now suppose that $A$ is a topological group. In each exact sequence $E: 0 \rightarrow A \stackrel{i}{\longrightarrow} B \stackrel{\pi}{\longrightarrow} G \longrightarrow 0$, require that $B$ be a topological group and $i$ and $\pi$ be continuous. By topological isomorphism (or equivalence) of two such sequences, we mean an isomorphism (or equivalence) $(\gamma, \beta, \phi)$ with all three maps homomorphisms. Let $\overline{\operatorname{Ext}}(G, A)$ be the resulting set of topological equivalence classes, and let $\operatorname{Ext}(G, A) \subset \overline{\operatorname{Ext}}(G, A)$ be the classes of those sequences $E: 0 \rightarrow A \stackrel{i}{\longrightarrow} B \stackrel{\pi}{\longrightarrow} G \rightarrow 0$ in which $\pi$ has a Borel right inverse, continuous in a neighborhood of the identity (it is easily seen that this property depends only on the equivalence class of a sequence).

Proposition 2.6. (Calabi). If $A$ is a topological group, and $\tau \epsilon$ $Z^{2}(G, A)$, then $B_{z}$ may be given a topology in such a way that $E_{-}$is a topological sequence, and $\left[E_{\text {: }}\right] \in \operatorname{Ext}(G, A)$. The map $\tau \rightarrow\left[E_{\text {: }}\right]$ is a bijection between $Z^{2}(G, A)$ and $\operatorname{Ext}(G, A)$.

Proof. See [5].

Proposition 2.7. Let $\tau_{k} \in Z^{2}\left(G_{k}, A_{k}\right), k=1,2 . \quad\left(A_{k}\right.$ topological groups.) Then $E_{\tau_{1}}$ is isomorphic with $E_{\tau_{2}}$ if and only if $\left[\tau_{1}\right]=\left[\tau_{2}\right]_{s}$ 
in $H^{2}\left(G_{1}, A_{1}\right)$ for some pair $s=(\phi, \gamma)$; where $\gamma: A_{1} \rightarrow A_{2}, \phi: G_{1} \rightarrow G_{2}$ are isomorphisms.

Proof. Suppose that $E_{\tau_{1}} \cong E_{\tau_{2}}$. There are isomorphisms $\gamma: A_{1} \rightarrow$ $A_{2}, \beta: B_{\tau_{1}} \rightarrow B_{\tau_{2}}, \phi: G_{1} \rightarrow G_{2}$ making the following diagram commutative:

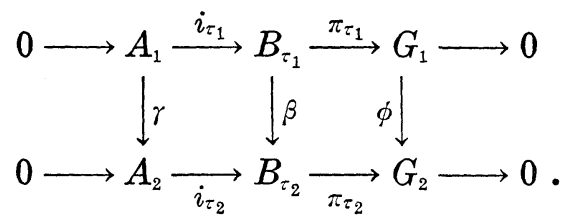

We recall that, as sets, $B_{\tau_{k}}=A_{k} \times G_{k}(k=1,2)$, and we define a map $p: G_{1} \rightarrow A_{2}$ by the equation:

$$
\beta(e, g)=(p(g), \phi(g)) .
$$

Let $\zeta=\gamma^{-1} p$. Then $\zeta \in F\left(G_{1}, A_{1}\right)$, and a computation, using the multiplicativity of $\beta$ and the definition of multiplication in $B_{\tau_{1}}$ and $B_{\tau_{2}}$, shows that $\zeta$ gives an equivalence between $\tau_{1}$ and $s \cdot \tau_{2}$, thus if $s=(\phi, \gamma)$, $\left[\tau_{1}\right]=s \cdot\left[\tau_{2}\right]$.

Conversely if $\left[\tau_{1}\right]=s \cdot\left[\tau_{2}\right]$ for $s=(\phi, \gamma)$ as above, then $\exists \zeta \in F\left(G_{1}\right.$, $A_{1}$ ) such that $\tau_{1}=\zeta \cdot\left(s \cdot \tau_{2}\right)$. If we then define $\beta: B_{\tau_{1}} \rightarrow B_{\tau_{2}}$ by:

$$
\beta(\alpha, x)=(\gamma(a \zeta(x)), \phi(x)),
$$

and use the definitions of $s \cdot \tau_{2}$ and $\zeta \cdot\left(s \cdot \tau_{2}\right)$, we can show that $\beta$ is an algebraic isomorphism. The local continuity of $\zeta$ shows that $\beta$ is continuous at the identity, and thus everywhere. This map, with $\phi$ and $\gamma$, impliments an isomorphism of $E_{\tau_{1}}$ with $E_{\tau_{2}}$. We omit the computations.

3. Vector valued measures. We begin with a discussion of vector valued measures as developed in [8]. We will use the second countability assumptions we have made to simplify certain points and we will make some slight changes in definitions. Throughout this section, $G$ is a fixed locally compact group. Let $B(G)$ be the $\sigma$-algebra of Borel sets in $G$ and $X$ be a separable Banach space.

Definition 3.1. A regular Borel measure on $G$ with values in $X$ is a map $m: B(G) \rightarrow X$ such that:

(i) $m\left(\bigcup_{i=1}^{\infty} B_{i}\right)=\sum_{i=1}^{\infty} m\left(B_{i}\right)$ (in norm) for every sequence $\left(B_{i}\right)_{1 \leqq i<\infty}$ of mutually disjoint sets in $B(G)$.

(ii) For every $\varepsilon>0$ and $B \in B(G), \exists$ a compact set $K \subset B$ and an open set $U \supset B$ such that $\forall B^{1} \in B(G)$ with $K \subset B^{1} \subset U, \| m(B)-$ $m\left(B^{1}\right) \|<\varepsilon$. 
DEFINITION 3.2. (a) Let $m$ be an $X$-valued regular Borel measure on $G$. The variation of $m$ is defined to be the positive regular Borel measure $|m|$ defined by $|m|(B)=\sup \left\{\sum_{i \in I}\left\|m\left(B_{i}\right)\right\|\right\}$ where the supremum is taken over all families $\left(B_{i}\right)_{i \in I}$ of mutually disjoint Borel sets contained in $B$.

(b) If $|m|(G)<+\infty$, we say that $m$ is of bounded variation.

The above definitions are essentially those contained in Dinculeanu [8], but his Borel measures are defined only on relatively compact Borel sets, and he defines finite variation (not bounded variation) to mean $|m|(B)<+\infty$ for all relatively compact Borel sets $B$.

Suppose now $E$ and $F$ are Banach spaces, and $\mathscr{L}(E, F)$ is the Banach algebra of all bounded linear operators from $E$ to $F$ with the usual norm and operations. Let $m$ be a regular Borel measure on $G$ taking values in $\mathscr{L}(E, F)$. We will always let $\mathscr{S}(G, E), C_{\infty}(G, E)$, $K(G, E)$ represent respectively the set of all $E$ valued functions on $G$ which are Borel measurable and have finite range, the continuous $E$-valued functions on $G$ vanishing at infinity, and the continuous $E$-valued functions on $G$ with compact support. When $E$ is the complex numbers, $C$, we will suppress it in the above notation.

DeFinition 3.3. (a) If $f=\sum_{n=1}^{N} x_{n} \chi_{B_{n}}$ belongs to $\mathscr{S}(G, E)(\chi$ represents a characteristic function), then $\int_{G} f d m$ is by definition

$$
\sum_{n=1}^{N} m\left(B_{n}\right) \cdot x_{n} \text { and }\|f\|_{1} \text { is } \sum_{n=1}^{N}\left\|x_{n}\right\||m|\left(B_{n}\right) \text {. }
$$

(b) If $f: G \rightarrow E$, we say $f$ is $m$-integrable if there is a $\|\cdot\|_{1-}$ Cauchy sequence $\left(f_{n}\right)_{1 \leqq n<\infty}$, in $\mathscr{S}(G, E)$ which converges $|m|$-almost everywhere to $f$.

The following list of facts can be deduced from or found in [8]. Some of them make use of the countability assumptions which we have in force. In the future we will make free use of them without further mention.

Proposition 3.4. Let $f: G \rightarrow E$ and $m$ be a regular Borel measure on $G$ with values in $\mathscr{L}(E, F)$ and bounded variation $|m|$. Then:

(a) If $f$ is m-integrable and $\left(f_{n}\right)_{1 \leqq n<\infty}$ is a $\|\cdot\|_{1}$ Cauchy sequence in $\mathscr{S}(G, E)$, converging $|m|$-almost everywhere to $f$, then sequences $\left(\left\|f_{n}\right\|_{1}\right)_{1 \leqq n<\infty}$ and $\left(\int_{n} f_{n} d m\right)_{1 \leqq n<\infty}$ converge in $R$ and $F$ respectively, and we denote the limits by $\|f\|_{1}$ and $\int f d m$. These limits are independent of the sequence $\left(f_{n}\right)_{1 \leqq n<\infty}$.

(b) The space $L^{1}(m, E)$ of E-valued m-integrable functions is a 
Banach space with respect to $\|\cdot\|_{1}$. Furthermore

$$
\|f\|_{1}=\int_{G}\|f(x)\| d|m|(x) .
$$

The map $f \rightarrow \int f d m$ is continuous, and in fact

$$
\left\|\int f d m\right\| \leqq \int\|f(x)\| d|m|(x) \equiv\|f\|_{1} .
$$

(c) $f \in L^{1}(m, E) \Longleftrightarrow$ the map $x \rightarrow\|f(x)\|$ is in $L^{1}(|m|)$ and $f$ is $|m|$-measurable, i.e., $f^{-1}(B)$ is $|m|$-measurable for all Borel sets $B$ in $E$. Since $|m|$ is a regular, positive Borel measure, we can (and always will) regard $L^{1}(m, E)$ as the set of all Borel-measurable functions $f$ from $G$ to $E$ such that $\|f(x)\|$ is $|m|$-integrable.

(d) If $\left(f_{n}\right)$ is Cauchy in $L^{1}(m, E)$ and converges $|m|$-almost everywhere to $f \in L^{1}(m, E)$, then $f_{n}$ converges to $f$ in $L^{1}(m, E)$. Also, if $\left(f_{n}\right)$ is any sequence in $L^{1}(m, E)$ converging in that space to $f$, then some subsequence converges to $f,|m|$-almost everywhere.

(e) The obvious extension of the Lebesgue dominated convergence theorem holds.

(f) $K(G, E)$ is dense in $L^{1}(m, E)$, and the mapping $f \rightarrow \int_{G} f d m$ from $K(G, E)$ to $F$ is continuous when $K(G, E)$ has the supremum norm.

Corollary 3.5. Any norm bounded Borel measurable function $f$ from $G$ to $E$ is $m$-integrable. Also, if $g$ is continuous, and bounded from $G$ to $R^{+}$, and $\|f(x)\| \leqq g(x) \forall x \in G$, then $f$ is the $\|\cdot\|_{1}$ limit of a sequence $\left(f_{n}\right)_{1 \leqq n<\infty}$ in $K(G, E)$ such that for all $n$ and $x,\left\|f_{n}(x)\right\| \leqq$ $g(x)$.

Proof. Since $|m|(G)<+\infty$, (c) above shows that $f \in L^{1}(m, E)$. By $(f)$ and (d) there is a sequence $\left(f_{n}\right)$ in $K(G, E)$ converging to $f$ $|m|$-almost everywhere. If

$$
g_{n}(x)=\left\{\begin{array}{rr}
\min \left(1, g(x) /\left\|f_{n}(x)\right\|\right) \cdot f_{n}(x) \mid f_{n}(x) \neq 0 \\
0 & \mid f_{n}(x)=0,
\end{array}\right.
$$

then $g_{n} \in K(G, E) \forall n$ and $g_{n}(x) \rightarrow f(x)|m|$-almost everywhere. Since $\left\|g_{n}(x)\right\| \leqq|g(x)|$, (e) shows that $g_{n}$ converges to $f$ in $L^{1}(m, E)$ (noting that $g$ is $|m|$-integrable).

The measures described above can be characterized by the properties they exhibit as bounded linear maps from $K(G, E)$ to $F$, i.e., we have a vector valued "Riesz representation theorem". Let $L$ be 
a linear map from $K(G, E)$ to $F$ and let $B_{0}(G)$ be the relatively compact Borel sets in $G$. For each $S \in B(G)$ let $\left\|L_{S}\right\|==\sup \left\{\sum_{i}\left\|L\left(f_{i}\right)\right\|\right\}$ where the supremum is taken over all finite families $\left(f_{i}\right)$ in $K(G, E)$ such that $\sum_{i}\left\|f_{i}(x)\right\| \leqq \chi_{S}(x) \forall x \in G$.

Definition 3.6. We say that a map $L$ as above is dominated if | $\left|L_{S} \|\right| \mid<\forall S \in B_{0}(G)$.

This definition is due to Dinculeanu [8].

Theorem 3.7. (Dinculeanu [8], III, §19, Theorem 2.).

There exists an isomorphism between the set of all regular Borel measures $m$ with values in $\mathscr{L}(E, F)$, satisfying the condition $|m|(S)<$ $\infty \forall S \in B_{0}(G)$, and the set of dominated linear maps from $K(G, E)$ to $F$. If $m$ is such a measure, the corresponding linear map $L$ is given by:

$$
L(f)=\int_{G} f d m, f \in K(G, E) .
$$

Furthermore, if $U$ is open in $G$ then $\|\left|L_{U}\right||=| m \mid(U)$.

CoRollary 3.8. The above correspondence restricts to a one-to-one correspondence between the regular Borel measures $m$ of bounded variation on $G$ and with values in $\mathscr{C}(E, F)$, and linear maps $L$ from $K(G, E)$ to $F$ with $\left\|L_{G}\right\|<+\infty$. We will let $\|L\|=\left\|L_{G}\right\|$ and note that if $L \leftrightarrow m$, then $\|L\|=\|m\| \equiv|m|(G)$.

We now specialize to the case where $A$ is a Banach algebra and $E=F \equiv A$. We know that $\mathscr{C}_{L}(A)$ is isometrically embedded in $\mathscr{L}(A, A) \equiv \mathscr{L}(A)$, and we will regard $\mathscr{L}_{L}(A)$ as a subset of $\mathscr{L}(A)$. Both $\mathscr{C}_{L}(A)$ and $K(G, A)$ are right $A$ modules (if $m \in \mathscr{C}_{L}(A), f \in$ $K(G, A), a, b \in A$, then $(m a)(b) \equiv m(a b)$ and $(f a)(x)=(f(x) a), x \in G)$. We show now that the $A$-linear maps from $K(G, A)$ to $A$ are the ones of interest.

THEOREM 3.9. The correspondence of Theorem 3.7 restricts to give a bijection between the set of all regular, $\mathscr{L}_{L}(A)$-valued measures of bounded variation on $G$ and the set of right $A$-linear maps $L$ from $K(G, A)$ to $A$ with $\|L\|<+\infty$.

Proof. Suppose that $m: B(G) \rightarrow \mathscr{C}_{L}(A)$ is a regular Borel measure of bounded variation. If $f=\sum_{i=1}^{n} a_{n} \chi_{B_{n}}$ is in $\mathscr{S}(G, A)$, and $b \in A$, then

$$
\begin{aligned}
{\left[\int_{G} f(x) d m(x)\right] \cdot b } & =\left[\sum_{i=1}^{n} m\left(B_{n}\right) \cdot a_{n}\right] b=\sum_{i=1}^{n} m\left(B_{n}\right)\left(a_{n} b\right) \\
& =\int_{G}\left(\sum_{i=1}^{n}\left(a_{n} b\right) \chi_{B_{n}}(x)\right) d m(x)=\int_{G}(f \cdot b)(x) d m(x) .
\end{aligned}
$$


This relation will hold when we pass to the limit, thus $L(f)=\int_{G} f d m$ is right $A$-linear on $K(G, A)$, and $\|L\|<+\infty$.

Conversely suppose $L: K(G, A) \rightarrow A$ is right $A$-linear and $\|L\|<+\infty$. We know by Corollary 3.8 that $L(f)=\int_{G} f(x) d m(x)$, for some $\mathscr{L}(A)$-valued regular Borel measure $m$ with bounded variation, and $|m|(G)=\|L\|$. Then $\int_{G}(f(x) a) d m(x)=\left(\int_{G} f(x) d m(x)\right) a \forall f \in K(G, A)$ and $a \in A$. Now if $B \in B(G), b \in A$, we can approximate $b \chi_{B}$ (in $\|\cdot\|_{1}$ ) by a sequence $f_{n}$ in $K(G, A)$. It is not hard to show that $\left(f_{n} \cdot a\right)$ converges to $b a \chi_{B}$ and the above relation on integrals holds in the limit to give us that $m(B)(b a)=\int_{G} b a \chi_{B} d m=\left(\int_{G} b \chi_{B} d m\right) a=(m(B) b) a$ for all $a$, $b \in A$. Thus $m$ has values in $\mathscr{A}_{L}(A)$ and we are done.

Now let $A$ be a Banach algebra, and $\tau=(T, \alpha) \in Z^{2}(G, A)$. Let $\mathscr{A}_{L}(G, A)$ be the linear space of all regular, $\mathscr{A}_{L}(A)$-valued, Borel measures on $G$ with bounded variation. $\mathscr{A}_{L}(G, A)$ can, by the previous result, be identified with the $A$-linear functions $F$ from $K(G, A)$ to $A$ with $\|F\|<\infty$. It is a reasonably straightforward task to show that if $\left(F_{n}\right)$ is a Cauchy sequence of such functions, $F_{n}$ converges pointwise on $K(G, A)$ to an $A$-linear map $F$ and $F$ has finite norm. We omit this calculation. Thus we may regard $\mathscr{A}_{L}(G, A)$ as a Banach space with $\|m\|=|m|(G)=\left\|L_{m}\right\|, m \in \mathscr{C}_{L}(G, A)$.

Now if $m \in \mathscr{A}_{L}(G, A), x \in G$, we define $(x \cdot m)(S)$ to be $T(x)(m(S))$ for all $S \in B(G)$. Then

Proposition 3.10. $x \cdot m \in \mathscr{A}_{L}(G, A)$ and $\|x \cdot m\|=\|m\|$. The proof is straightforward and we omit it.

If $\mu, \nu$ are in $\mathscr{M}_{L}(G, A)$, we define a linear function $L(\mu, \nu)$ from $K(G, A)$ to $A$ as follows:

$$
L(\mu, \nu)(h)=\int_{G} \int_{G} \alpha(x, y) h(x y) d(x \cdot \nu)(y) d \mu(x) .
$$

Lemma 3.11. $L(\mu, \nu)$ is defined and is a right A-linear function with $\|L\|<\infty$. Moreover, $\|L(\mu, \nu)\| \leqq\|\mu\|\|\nu\|$.

Proof. For $a \in A$, the function $(x, y) \rightarrow \alpha(x, y) a$ from $G \times G$ to $A$ is Borel measurable. It follows easily that if $h \in K(G, A)$, the function $k(x, y)=\alpha(x, y) h(x y)$ is Borel measurable and norm bounded. Thus $\int_{G} \alpha(x, y) h(x y) d(x \cdot \nu)(y) \equiv g(x)$ exists by Corollary 3.5. Furthermore, separability of $A$ and second countability of $G$ allow us to approximate $k(x, y)$ uniformly by functions of the form $\sum_{n=1}^{\infty} a_{n} \chi_{c_{n}}(x) \chi_{D_{n}}(y)$ 
where $C_{n}, D_{n}$ are in $B(G)$, and $a_{n} \in A$. The $(x \cdot \nu)$-integral of such a function in $y$ is $\sum_{n=1}^{\infty} \chi_{c_{n}}(x) T(x)\left(\nu\left(D_{n}\right)\right) a_{n}$ which is Borel measurable by Lemma 2.2, and converges pointwise in $x$ to $g(x)$. Thus $g(x)$ is Borel measurable and since $\|g(x)\| \leqq\|h\|_{\infty}\|x \cdot \nu\|=\|h\|_{\infty}\|\nu\|<\infty$, Corollary 3.5 implies that $L(\mu, \nu)$ exists. It is trivial that $L(\mu, \nu)$ is $A$-linear. We now show that $\|L(\mu, \nu)\| \leqq\|\mu\|\|\nu\|<\infty$.

Choose a finite set $f_{1}, \cdots, f_{p}$ of functions in $K(G, A)$ such that $\forall x \in G, \sum_{i=1}^{p}\left\|f_{i}(x)\right\| \leqq 1$. Let $f_{i}^{x}(y) \equiv f_{i}(x y)$. Then for any fixed $x, g_{i, x}(y) \equiv$ $\alpha(x, y) f_{i}(x y)$ is bounded in norm by $\left\|f_{i}^{x}(y)\right\|$ (a continuous function) and is Borel measurable. By Corollary 3.5, we can choose, for each $i, x$, a sequence $\left(g_{i, x}^{n}\right)_{1 \leqq n<\infty}$ in $K(G, A)$ such that $\left\|g_{i, x}^{n}(y)\right\| \leqq\left\|f_{i}^{x}(y)\right\| \forall y \epsilon$ $G$, and $g_{i, x}^{n}$ converges in $L^{1}(x \cdot \nu)$ to $g_{i, x}$ as $n \rightarrow \infty$. Then for any $n$,

$$
\sum_{i=1}^{p}\left\|\int_{G} g_{i, x}^{n}(y) d(x \cdot \nu)(y)\right\| \leqq\|x \cdot \nu\|=\|\nu\|
$$

(Proposition 3.10), since

$$
\sum_{i=1}^{p}\left\|g_{i, x}^{n}(y)\right\| \leqq \sum_{i=1}^{p}\left\|f_{i}^{x}(y)\right\| \leqq 1 \quad \forall y \in G .
$$

A limiting argument then shows that

$$
\begin{aligned}
& \sum_{i=1}^{p}\left\|L(\mu, \nu)\left(f_{i}\right)\right\|=\sum_{i=1}^{p}\left\|\int_{G} \int_{G} \alpha(x, y) f_{i}(x y) d(x \cdot \nu)(y) d \mu(x)\right\| \\
\leqq & \|\mu\| \sup _{x}\left(\sum_{i=1}^{p}\left\|\int_{G} g_{i, x}(y) d(x \cdot \nu)(y)\right\|\right) \leqq\|\mu\|\|\nu\| .
\end{aligned}
$$

Thus

$$
\|L(\mu, \nu)\| \leqq\|\mu\|\|\nu\|<+\infty .
$$

Definition 3.12. The $\tau$-twisted convolution $\mu * \nu$, of $\mu$ and $\nu$ is the measure in $\mathscr{A}_{L}(G, A)$ corresponding to the functional $L(\mu, \nu)$.

We will now prove that $\mathscr{M}_{L}(G, A)$ is a Banach algebra with $\tau$ twisted convolution, for any $\tau \in Z^{2}(G, A)$. The only thing missing is the fact the $*$ is associative. We first need some Lemmas.

Lemma 3.13. If $h \in K(G, A), \mu \in \mathscr{C}_{L}(G, A), z \in G$, then

$$
z \cdot\left[\int_{G} h d \mu\right]=\int_{G}(z \cdot h(x)) d(z \cdot \mu)(x) \text {. }
$$

Proof. Let $f=\sum_{k=1}^{n} a_{k} \chi_{B_{k}} \in \mathscr{S}(G, A)$. Then

$$
\begin{aligned}
& z \cdot \int_{G} f(x) d \mu(x)=z \cdot\left(\sum_{k=1}^{n} \mu\left(B_{k}\right) a_{k}\right)=\sum_{k=1}^{n}\left(z \cdot \mu\left(B_{k}\right)\right)\left(z \cdot a_{k}\right) \\
= & \int_{G} \sum_{k=1}^{n}\left(z \cdot a_{k}\right) \chi_{B_{k}} d(z \cdot \mu)=\int_{G}(z \cdot f)(x) d(z \cdot \mu)(x) .
\end{aligned}
$$


The result follows by approximating $h$ with functions in $\mathscr{S}(G, A)$.

Lemma 3.14. If $h \in K(G, A), \nu, \mu \in \mathscr{M}_{L}(G, A)$, and $z \in G$, then

$$
\int_{G} h(x) d(z \cdot(\mu * \nu))(x)=\int_{G} \int_{G}(z \cdot \alpha(s, t)) h(s t) d(z \cdot(s \cdot \nu))(t) d(z \cdot \mu)(s) \cdot
$$

Proof.

$$
\begin{aligned}
& \int_{G} h(x) d(z \cdot(\mu * \nu))(x)=z \cdot \int_{G}\left(T(z)^{-1} h(x)\right) d(\mu * \nu)(x) \text { (by Lemma 3.13) } \\
= & z \cdot\left[\int_{G} \int_{G} \alpha(s, t)\left[T(z)^{-1} h(s t)\right] d(s \cdot \nu)(t) d \mu(s)\right. \\
= & \iint(z \cdot \alpha(s, t)) h(s t) d(z \cdot(s \cdot \nu))(t) d(z \cdot \mu)(s) .
\end{aligned}
$$

THEOREM 3.15. The multiplication * on $\mathscr{A}_{L}(G, A)$ (with respect to some given $\tau=(T, \alpha)$ ) is associative.

Proof. Let $\nu, \mu, \gamma \in \mathscr{M}_{L}(G, A), h \in K(G, A)$. Then $[\nu *(\mu * \gamma)](h)$

$$
\begin{aligned}
& =\int_{G} \int_{G} \alpha(z, \omega) h(z \omega) d(z \cdot(\mu * \gamma))(\omega) d \nu(z) \\
& =\int_{G} \int_{G} \int_{G}(z \cdot \alpha(s, t)) \alpha(z, s t) h(z s t) d(z \cdot(s \cdot \gamma))(t) d(z \cdot \mu)(s) d \nu(z) \\
& =\int_{G} \int_{G} \alpha(z, s) \int_{G} \alpha(z s, t) h(z s t) d(z s \cdot \gamma)(t) d(z \cdot \mu)(s) d \nu(z) \\
& \text { (by Def. 2.1-a, (ii) and (iii)) } \\
& =\int_{G} \int_{G} \alpha(x, t) h(x t) d(x \cdot \gamma)(t) d(\mu * \nu)(x)=[(\mu * \nu) * \gamma](h) .
\end{aligned}
$$

We thus have proved that $\mathscr{M}_{L}(G, A)$, with multiplication *, is a Banach algebra which we will denote $\mathscr{M}_{L}(G, A ; \tau)$.

4. Centralizers on twisted group algebras.

Definition 4.1. Let $G$ be a locally compact group, $A$ a Banach *algebra and $\tau=(T, \alpha) \in Z^{2}(G, A)$. Let $L^{1}(G, A ; \tau)$ be the Banach *-algebra consisting of all Bochner integrable functions from $G$ to $A$ with multiplication given by:

$$
(f \circ g)(x)=\int_{G} f(y)\left(T(y) g\left(y^{-1} x\right)\right) \alpha\left(y, y^{-1} x\right) d y
$$

and involution given by:

$$
f^{*}(x)=\alpha\left(x, x^{-1}\right)^{*}\left(T(x) f\left(x^{-1}\right)^{*} \Delta(x)^{1 / 2}\right.
$$


where $\Delta$ is the modular function for $G$.

The fact that this is a Banach *-algebra can be found in [4]. If $f \in L^{1}(G, A ; \tau)$, let $L_{f}: K(G, A) \rightarrow A$ be given by:

$$
L_{f}(h)=\int_{G} f(x) h(x) d x \text { for } h \in K(G, A) .
$$

Clearly $L_{f}$ is linear. If $a \in A$ then

$$
L_{f}(h \cdot a)=\int_{G} f(x)(h(x) a) d x=\left(\int_{G} f(x) h(x) d x\right) a=L_{f}(h) \cdot a,
$$

so $L_{f}$ is right $A$-linear. Finally if $\left(f_{i}\right)_{1 \leqq i \leqq n}$ is a finite family from $K(G, A)$ such that $\forall x \in G$,

$$
\sum_{i=1}^{n}\left\|f_{i}(x)\right\| \leqq 1
$$

then

$$
\begin{aligned}
\sum_{i=1}^{n}\left\|L_{f}\left(f_{i}\right)\right\| & =\sum_{i=1}^{n}\left\|\int_{G} f(x) f_{i}(x) d x\right\| \leqq\left(\sum_{i=1}\left\|f_{i}\right\|_{\infty}\right) \int_{G}\|f(x)\| d x \\
& \leqq\|f\|_{1}
\end{aligned}
$$

and so

$$
\left\|L_{f}\right\| \mid \leqq\|f\|_{1}<\infty
$$

Proposition 4.2. The above correspondence induces a map $\phi$ from $L^{1}(G, A ; \tau)$ to $\mathscr{M}_{L}(G, A ; \tau)$ such that $\phi$ is an isometric monomorphism, and $\phi\left(L^{1}(G, A ; \tau)\right)$ is a norm closed, left ideal in $\mathscr{A}_{L}(G, A ; \tau)$.

Proof. Let $\mu_{f}$ be the measure corresponding to $L_{f}$. Then $\mu_{f}(S)=$ $\int_{S} f(x) d x \forall S \in \mathscr{B}(G)$. The fact that $\phi$ is isometric follows from [8], Ch. II, $\S 10$, no. 2, and Ch. II, $\S 10$, no. 9, Th. 6 .

Now if $f, g \in L^{1}(G, A ; \tau)$, and $h \in K(G, A)$, an easy computation shows that $\left(\mu_{f} * \mu_{g}\right)(h)=\mu_{f^{*}}(h)$ and so $\phi$ preserves product. Since $L^{1}(G, A ; \tau)$ is complete and $\phi$ is isometric, $\phi\left(L^{1}(G, A ; \tau)\right)$ is norm closed. From now on we will always identify $f \in L^{1}(G, A ; \tau)$ with $\mu_{f} \in$ $\mathscr{A}_{L}(G, A ; \tau)$.

To show that $L^{1}(G, A ; \tau)$ is a left ideal in $\mathscr{M}_{L}(G, A ; \tau)$, let $f \in$ $K(G, A)$ and $m \in \mathscr{K}_{L}$. Let

$$
f_{m}(x)=\int_{G}\left(T(y) f\left(y^{-1} x\right)\right) \alpha\left(y, y^{-1} x\right) d m(y) .
$$

Since

$$
f \in K(G, A), \quad y \longrightarrow\left[T(y) f\left(y^{-1} x\right)\right] \alpha\left(y, y^{-1} x\right)
$$


is norm bounded and Borel measurable, thus $m$ integrable, and so $f_{m}(x)$ exists. Now

$$
\begin{aligned}
& \int_{G}\left\|f_{m}(x)\right\| d x=\int_{G}\left\|\int_{G}\left(T(y) f\left(y^{-1} x\right)\right) \alpha\left(y, y^{-1} x\right) d m(y)\right\| d x \\
\leqq & \int_{G} \int_{G}\left\|f\left(y^{-1}(x)\right)\right\| d x d|m|(y) \\
= & \|f\|_{1}\|m\|<+\infty, \text { so } f_{m} \in L^{1}(G, A ; \tau) .
\end{aligned}
$$

Now if $h \in K(G, A)$, then

$$
\begin{aligned}
\left(m * \mu_{f}\right)(h) & =\int_{G} \int_{G} \alpha(y, x) h(y x) d\left(y \cdot \mu_{f}\right)(x) d m(y) \\
& =\int_{G} \int_{G}[T(y) f(x)] \alpha(y, x) h(y x) d x d m(y) \\
& =\int_{G} \int_{G}\left[T(y) f\left(y^{-1} x\right)\right] \alpha\left(y, y^{-1} x\right) h(x) d x d m(y) \\
& =\int_{G}\left[\int_{G}\left[T(y) f\left(y^{-1} x\right)\right] \alpha\left(y, y^{-1} x\right) d m(y)\right] h(x) d x \\
& =\int_{G} f_{m}(x) h(x) d x=\mu_{f_{m}}(h) .
\end{aligned}
$$

The result follows for $f$ in $L^{1}(G, A ; \tau)$ by approximating with functions form $K(G, A)$. Hence $\phi\left(L^{1}(G, A ; \tau)\right)$ is a norm closed left ideal in $\mathscr{C}_{L}(G, A ; \tau)$.

We now propose to prove that $\mathscr{M}_{L}(G, A ; \tau)$ satisfies the two conditions of Proposition 1.5 relative to $L^{1}(G, A ; \tau)$ and so is isometrically isomorphic with the left centralizer algebra of $L^{1}(G, A ; \tau)$. This is our main result, and the proof is quite involved, largely due to the presence of a non-globally continuous cocycle $\alpha$. Our theorem specializes to a theorem of Wendel [21] when $A=C$ and $\tau$ is trivial, and our proof begins like a vector generalization of his, but his proof uses weak-*compactness of the measures of norm one, a property which does not generalize at all, so we proceed in a different way. Even in Wendel's case, our proof specializes to a proof different from his. We first prove some technical lemmas.

Lemma 4.3. Let $\tau \in Z^{2}(G, A), \tau \equiv(T, \alpha)$, and let $s \in G$. Then there exist:

(1) a relatively compact neighborhood $U(s)$ of $s$ and a set $M(s)$ whose complement is a compact set of measure zero in $G$ and,

(2) for each $t \in M(s)$, a relatively compact neighborhood $V(s, t)$ of $t$ and $a$ Borel function $p_{s, t}: G \rightarrow \mathscr{U}(A)$, both depending on $s$ and $t$, such that if $\left(T_{s, t}, \alpha_{s, t}\right)=p_{s, t} \cdot(T, \alpha)$, then: 
(i) $\alpha_{s, t}(x, y)$ is continuous for $x$ in $U(s)$ and $y$ in $V(s, t)$,

(ii) $T_{s, t}(x)$ is continuous for $x$ in $U(s) \cup V(s, t)$, (and $(x, a) \rightarrow$ $T_{s, t}(x) a$ is continuous in $\left.(U(s) \cup V(s, t)) \times A\right)$,

(iii) if $t_{1}, t_{2} \in M(s)$ then $p_{s, t_{1}}(x)=p_{s, t_{2}}(x) \forall x \in U(s)$, and

(iv) $e \in M(s) \forall s \in G$.

This is an extremely important technical lemma quite similar to the one proved in [2], but not a consequence of it. We will only sketch the proof.

Proof. As in [2], Lemma 2, we note that there is a base $\mathscr{S}$ of neighborhoods of $e$ consisting of sets $V$ such that:

(a) $V$ is open, symmetric, and relatively compact, and

(b) if $n$ is any positive integer then the boundary of $V^{n}$ has Haar measure zero.

Now we first suppose that $s \neq e$, and we pick $W$ from $\mathscr{S}$ such that

$$
W s W^{2} s^{-1} W \subset N, W^{2} \subset N_{s} \cap N_{s^{-1}} \text { and } s \notin W^{5} \text {. }
$$

Let $N(s)=\mathrm{bd}(W s) \cup \mathrm{bd}\left(s^{-1} W^{2} s\right)$ which is compact and of measure zero by hypothesis. Let $M(s)$ be the complement of $N(s)$, and let $t \in M(s)$.

Case 1. $t \notin W s \cup s^{-1} W^{2} s$ then $t \notin \overline{W s}$ and $t \notin \overline{s^{-1} W^{2} s}$, and so we can choose a symmetric neighborhood $V$ of $e$ such that

(i) $V t \cap W s=\phi$

(ii) $W s \cap W s V t=\phi$

(iii) $V \subset W$ (which implies $V t \cap W s V t=\phi$ since $s \notin W^{3}\left(\subset W^{5}\right)$ ).

Notice that (iii) also implies $V \subset N_{s^{-1}}$ and $s V s^{-1} W \subset N$.

Since $V t, W s, W s V t$ are disjoint we can define $p_{s, t}$ as follows: if $x=w s \in W s$ then $p_{s, t}(x)=\alpha(w, s)$. If $y=v t \in V t, p_{s, t}(y)=\alpha(v, t)$, and if $z=w s v t=\left(w s v s^{-1}\right) s t \in W s V t$ then $p_{s, t}(z)=\alpha\left(w s v s^{-1}, s t\right)$. Otherwise $p_{s, t} \equiv I$. Then if $x=w s \in W s$ and $y=v t \in V t$, a somewhat length computation shows that

$$
\alpha_{s, t}(x, y)=\left(T(w) \beta\left(s, v, s^{-1}\right)\right) \alpha\left(w, s v s^{-1}\right) T\left(w s v s^{-1}\right)\left[\alpha\left(s, s^{-1}\right)^{*} \alpha(s, t)\right] .
$$

Thus $\alpha_{s, t}$ is easily shown to be continuous on $W s \times V t$ by using the facts $V \subset N_{s^{-1}}, s V s^{-1} \subset N, W s V s^{-1} \subset N$.

We also have $T_{s, t}(x)=T(w) T(s)$ and $T_{s, t}(y)=T(v) T(t)$. Thus if we let $U(s)=W s$ and $V(s, t)=V t$, (i) and (ii) hold. 
Case 2. $t \in s^{-1} W^{2} s$. Since $s \notin W^{5}, W^{3} s \cap s^{-1} W^{2} s=\phi$. If $\bar{x}=\bar{w} s$ is in $W^{3} s$, let $p_{s, t}(\bar{x})=\alpha(\bar{w}, s)$ otherwise $p_{s, t} \equiv I$.

Now if $x=w s \in W s$ and $y=s^{-1} w^{1} s \in s^{-1} W^{2} s$ then:

$$
\alpha_{s, t}(x, y)=\left[T(w) T(s) \beta\left(s^{-1}, w^{1}, s\right)^{*}\right]\left[T(w) T(s) \alpha\left(s^{-1}, s\right)\right] \alpha\left(w, w^{1}\right),
$$

and the facts; $W^{2} \subset N_{s}, W^{2} \subset N$, prove that the above is continuous on $W s \times s^{-1} W^{2} s$. Also

$$
T_{s, t}(x)=T(w) T(s) \text { and } T_{s, t}(y)=T(y) .
$$

Thus if we let $U(s)=W s$ and $V(s, t)=s^{-1} W s$, (i) and (ii) are true.

Case 3. Finally suppose $t \in W s$. Since $s \notin W^{3}, W s \cap W s W s=\phi$. If $u=w s \in W s$, let $p_{s, t}(u)=\alpha(w, s)$. If $v=w s w^{1} s=w s w^{1} s^{-1} s^{2} \in W s W s$,

$$
p_{s, t}(v)=\alpha\left(w s w^{1} s^{-1}, s^{2}\right) \text {. Otherwise } p_{s, t} \equiv I .
$$

Now suppose $x=w s$ and $y=w^{1} s$ are both $W s$. Then

$$
\alpha_{s, t}(x, y)=\left(T(w) \beta\left(s, w^{1}, s^{-1}\right)\right) \alpha\left(w, s w^{1} s^{-1}\right) T\left(w s w^{1} s^{-1}\right)\left(\alpha\left(s, s^{-1}\right)^{*} \alpha(s, s)\right) \text {. }
$$

Once again we have continuity due to the facts:

$$
\begin{aligned}
& W \subset N_{s^{-1}}, W \subset N, s W s^{-1} \subset N, \\
& W s W s^{-1} \subset N .
\end{aligned}
$$

Finally $T_{s, t}(x)=T(w) T(s)$ and $T_{s, t}(y)=T\left(w^{1}\right) T(s)$, so again (i) and (ii) hold if $U(s)=W s$ and $V(s, t)=W s$. In all three cases, if $x=w s \epsilon$ $U(s) \equiv W s$ then $p_{s, t}(x)=\alpha(w, s)$ regardless of $t$, so (iii) holds, and finally (iv) holds by choice of $W$. Similar (but simpler) considerations hold when $s=e$.

For future reference the operators $h \rightarrow L_{f}(h)$ on $K(G, A)$ ( $f$ in $L^{\prime}(G, A)$ ) will be denoted $h \rightarrow f(h)$.

Lemma 4.4. Let $h \in K(G, A), \varepsilon>0$ be given. Then $\exists f \in K(G) \equiv$ $K(G, C), f \geqq 0$, such that

$$
\left\|\int_{G} f(y)[h(x y)-h(x)] d y\right\|<\varepsilon \forall x \in G, \text { and } \int_{G} f(y) d y=1 .
$$

Proof. $h$ is uniformly continuous on $G$, so there is a relatively compact neighborhood $U$ of $e$ such that if $y \in U,\|h(x y)-h(x)\|<\varepsilon$. Pick $f \geqq 0$ with support in $U$ such that $\int_{G} f(y) d y=1$. Then 


$$
\begin{aligned}
& \left\|\int_{G} f(y)[h(x y)-h(x)] d y\right\| \\
= & \left\|\int_{U} f(y)[h(x y)-h(x)] d y\right\|<\varepsilon \int_{U} f(y) d y=\varepsilon .
\end{aligned}
$$

Lemma 4.5. Let $s \in G$ and $h \in K(G, A)$. Then there is a neighborhood $V$ of $e$ such if $y \in V, x \in U(s), z \in G$,

$$
\left\|\alpha_{s, e}(x, y) h(z)-h(z)\right\|<\varepsilon .
$$

Proof. $e \in M(s)$ and so $\alpha_{s, e}$ is continuous on $U(s) \times V(s, e)$. Since $\alpha_{s, e}(x, e)=I$ (Case 2) for all $x$ in $U(s)$, the result follows from the continuity of $\alpha_{s, e}$ if $V \subset V(s, e)$ is chosen small enough (note that $h(G)$ is compact in $A$ ).

LemmA 4.6. If $\left(k_{n}\right)_{1 \leqq n<\infty}$ is a Cauchy sequence in $L^{1}(G, A ; \tau)$ and $\forall h \in K(G, A), k_{n}(h) \rightarrow \bar{k}(h)$, then $k_{n} \rightarrow \bar{k}$ in $L^{1}(G, A ; \tau)$.

Proof. The lemma is easily seen to be a consequence of the following result: If $f \neq 0$ is in $L^{1}(G, A)$ then there exists an $h$ in $K(G, A)$ with $\int_{G} f(x) h(x) d x \equiv f(h) \neq 0$. We prove this general result.

Since $f$ is not zero in $L^{1}(G, A)$, there exists a compact subset $K$ in $G$ such that (i) $|K \cap U|=0(|S|=$ Haar measure of $S)$ implies $K \cap U=\phi$ for every open subset $U$ of $G$, (ii) $f_{0} \equiv f \mid K$ is continuous on $K$ and (iii) $\left\|f_{0}(x)\right\|>0$ on $K$. Take a fixed $z$ in $K$. Since $f(z) \neq 0$ there is an element $a$ in $A$ with $f(z) a \neq 0$. Then $K_{1}=\{x \in K \mid\|f(x) a\|>0\}$ is a neighborhood of $z$ in $K$, and so $\left|K_{1}\right|>0$. Let $\varphi$ be a real valued continuous linear functional on $A$ with $\varphi(f(z) a)=1$. It follows that $x \rightarrow \varphi(f(x) a)$ is integrable on $G$, continuous and nonzero on $K_{1}$ and consequently nonzero in $L^{1}(G)$. Thus there exists $u \in K(G)$ with

$$
\int_{G} \varphi(f(x) a) u(x) d x=\int_{G} \varphi(f(x) h(x)) d x=\varphi\left(\int_{G} f(x) h(x) d x\right) \neq 0,
$$

with $h(x)=a u(x), h \in K(G, A)$.

LEMMA 4.7. Let $\left(e_{n}\right)_{1 \leqq n<\infty}$ be an approximate identity in $A$, and $T_{0} \in \mathscr{A}(A)$. Then $T_{0}\left(e_{n}\right) \rightarrow I$ strongly as $n \rightarrow \infty$.

Proof. Let $a \in A$. Then $T_{0}\left(e_{n}\right) a=T_{0}\left(e_{n}\left[T_{0}^{-1}(a)\right]\right)$. Thus $\left\|T_{0}\left(e_{n}\right) a-a\right\|$ $\left.=\left\|T_{0}\left(e_{n}\left[T_{0}^{-1}(a)\right]-T_{0}^{-1}(a)\right)\right\| \leqq \| e_{n}\left[T_{0}^{-1}(a)\right]-T_{0}^{-1}(a)\right) \| \rightarrow 0$ as $n \rightarrow \infty$.

We now begin a sequence of lemmas leading to the proof that 
$\mathscr{A}_{L}(G, A ; \tau)$ is isometrically isomorphic with $\mathscr{C}_{L}\left(L^{1}(G, A ; \tau)\right)$.

For each $s \in G$, let $W(s)$ be a neighborhood of $s$ such that $\overline{W(s)} \subset$ $U(s)$.

LEMmA 4.8. Let $\left(f_{n}\right)_{1 \leqq n \leq \infty}$ be a sequence in $L^{1}(G, A ; \tau)$ such that:

(a) $\left(f_{n}\right)$ is strongly Cauchy,

(b) $\left\|f_{n}\right\|_{1} \leqq 1$ for all $n$,

(c) $\operatorname{supp}\left(f_{n}\right) \subseteq W(s)$ for all $n$ (fixed $s$ ).

Then $\exists m \in \mathscr{A}_{L}(G, A ; \tau)$ such that:

(1) $\operatorname{supp}(m) \subseteq W(s)$

(2) strong- $\lim _{n \rightarrow \infty} f_{n}=m$.

Furthermore, we have,

(3) $\|m\|=\sup _{f}\{\|m * f\|\}$ where $f$ ranges over the unit ball in $L^{1}(G, A ; \tau)$.

Proof. Choose $t \in M(s)$ and define $p: G \rightarrow \mathscr{U}(A)$ by;

$$
p(x)=\left\{\begin{array}{r}
p_{s, t}(x) \mid x \in W(s) \\
I \mid x \notin W(s) .
\end{array}\right.
$$

Lemma 4.3, (iii) tells us that $p$ does not depend on which $t \in M(s)$ was chosen. Let $g_{n}(x)=p(x) f_{n} \in L^{1}(G, A)$ for all $n$. Now let $h \in K(G, A)$, and $\varepsilon>0$ be given. By Lemmas $4.3,4.4$, and 4.5 , we can find an $f \in K(G)$ such that:

(i ) $f \geqq 0, \int_{G} f(x) d x=1, \operatorname{supp}(f) \subset V(s, e)$,

(ii) $\left\|\int_{G} f(y)[h(x y)-h(x)] d y\right\|<\varepsilon / 12$,

(iii) if $x \in W(s)$ and $y \in \operatorname{supp}(f)$,

$$
\left\|\alpha_{s, e}(x, y) h(x y)-h(x y)\right\|<\frac{\varepsilon}{12} .
$$

Let $I(x)=\left[\int_{G} f(y) \alpha_{s, e}(x, y) h(x y) d y-h(x)\right]$.

Then if

$$
\begin{aligned}
& x \in W(s),\|I(x)\| \\
\leqq & \left\|\int_{G} f(y)\left[\alpha_{s, e}(x, y) h(x y)-h(x y)\right] d y\right\|+\left\|\int_{G} f(y)[h(x y)-h(x)] d y\right\| \\
< & \frac{\varepsilon}{12}+\frac{\varepsilon}{12}=\frac{\varepsilon}{6} .
\end{aligned}
$$

Let $\left(e_{i}\right)_{1 \leqq i<\infty}$ be an approximate identity in $A$, and $F_{i}(x)=e_{i} f(x)$. Since $p_{s, e}$ is an isomorphism from $L^{1}(G, A ; \tau)$ onto $L^{1}\left(G, A ; \tau_{s, e}\right)$, and 
$p_{s, e} \equiv p$ on $W(s),\left(g_{n}\right)$ is strongly Cauchy in $L^{1}\left(G, A ; \tau_{s, e}\right)$ and so $\left(g_{n} * F_{i}\right)_{1 \leqq n<\infty}$ is norm Cauchy in $L^{1}\left(G, A ; \tau_{s, e}\right)$.

Thus $\left(\left(g_{n} * F_{i}\right)(h)\right)_{1 \leqq n<\infty}$ is Cauchy in $A$, and

$$
\begin{aligned}
& \left\|\left(g_{n} * F_{i}\right)(h)-g_{n}(h)\right\| \\
\leqq & \int_{G}\left\|g_{n}(x)\right\|\left\|\int_{G} T_{s, e}(x)\left(F_{i}(y)\right) \alpha_{s, e}(x, y) h(x y) d y-h(x)\right\| d x \\
< & \int\left\|g_{n}(x)\right\|\|I(x)\| d x+\int_{G}\left\|g_{n}(x)\right\|\left\|T_{s, e}(x)\left(e_{i}\right) h(x)-h(x)\right\| d x .
\end{aligned}
$$

Now Lemma 4.7 implies that for each $x \in G, \lim _{i \rightarrow \infty}\left\|T_{s, e}(x)\left(e_{i}\right) h(x)-h(x)\right\|=$ 0 and thus the Lebesgue theorem tells us that the second integral above tends to zero as $i \rightarrow \infty$. Since the integrand of the first integral above vanishes outside $W(s)$, we see that $\exists i_{0}$ such that if $i \geqq i_{0}$ then

$$
\left\|\left(g_{n} * F_{i}\right)(h)-g_{n}(h)\right\|<\varepsilon / 6+\varepsilon / 6=\varepsilon / 3,
$$

for all positive integers $n$.

The fact that $\left(\left(g_{n} * F_{i}\right)(h)\right)_{1 \leqq n<\infty}$ is Cauchy combines with $\left(^{*}\right)$ to show that there is an $n_{0}$ such that if $n, m \geqq n_{0},\left\|g_{n}(h)-g_{m}(h)\right\|<\varepsilon$. Thus, $\left(g_{n}(h)\right)_{1 \leqq n<\infty}$ is Cauchy and converges to an element $L(h) \in A$. The map $L: K(G, A) \rightarrow A$, defined in this way, is easily seen to be linear and $A$-linear, and since $\left\|g_{n}\right\| \leqq 1 \forall n$, it follows that $\|L\| \leqq 1$. $L$ then corresponds to a measure $\bar{m}$ which clearly has support in $W(s)$.

Now if we pass to the limit (in $n$ ) in the relation $\left({ }^{*}\right)$, we have $\left\|\left(\bar{m} * F_{i}\right)(h)-\bar{m}(h)\right\|<\varepsilon / 3$ if $i \geqq i_{0}$ (where $i_{0}$ depends on $h$ ). Since $\|\bar{m}\|$ can be approximated arbitrarily closely by sums $\sum_{j=1}^{s}\left\|\bar{m}\left(h_{j}\right)\right\|$, where $\sum_{j=1}^{s}\left\|h_{j}(x)\right\| \leqq 1$ for all $x$, the above relation shows that $\|\bar{m}\|$ can similarly approximated from below by sums $\sum_{j=1}^{s}\left\|\left(\bar{m} * F_{i}\right)\left(h_{j}\right)\right\|$. Thus

$$
\|\bar{m}\| \leqq \overline{\lim _{i}}\left\|\bar{m} * F_{i}\right\| \leqq\|\bar{m}\|
$$

and so

$$
\|\bar{m}\|=\sup _{\|f\| \leqq 1}\|\bar{m} * f\|
$$

were $f \in L^{1}(G, A)$.

Let $m$ be the measure $p^{-1} \cdot \bar{m}$, i.e.,

$$
m(S)=\int_{S} p^{-1}(x) d \bar{m}(x), S \in B(G) .
$$

We will now show that $\left(f_{n}\right)$ converges strongly to $m$ in $\mathscr{M}(G, A ; \tau)$. Let $t \in M(s)$ and suppose that $f \in K(G, A)$ has support in $V(s, t)$. Again $p \equiv p_{s, t}$ on $W(s)$, and $p_{s, t}$ gives an isomorphism of $L^{1}(G, A ; \tau)$ with $L^{1}\left(G, A ; \tau_{s, t}\right)$, so $\left(g_{n}\right)_{1 \leqq n<\infty}$ is strongly Cauchy in $L^{1}\left(G, A ; \tau_{s, t}\right)$.

Choose $\gamma \in K(G)$ with support in $U(s)$, so that $\gamma \equiv 1$ on $W(s)$. 
Then if

$$
h \in K(G, A),\left(g_{n} * f\right)(h)=\int_{G} g_{n}(x)\left[\int_{G} \gamma(x)\left(T_{s, t}(x)(f(y)) \alpha_{s, t}(x, y) h(x y) d y\right] d x\right.
$$

(since $\operatorname{supp}\left(g_{n}\right) \subset W(s) \forall n$ ), and our assumptions on $\gamma, T_{s, t}, \alpha_{s, t}$, and $f$ show that the inner integrand is zero outside $U(s) \times V(s, t)$ and continuous on this product. The inner integral as a function of $x$ belongs to $K(G, A)$ and thus the entire expression tends to

$$
\int_{G} \int_{G}\left(T_{s, t}(x)(f(y)) \alpha_{s, t}(x, y) h(x y) d y d \bar{m}(x)=(\bar{m} * f)(h)\right.
$$

(* in $\left.\mathscr{l}_{L}\left(G, A ; \tau_{s, t}\right)\right)$. Lemma 4.6 shows that

$$
\left(g_{n} * f\right) \longrightarrow \bar{m} * f \text { in } L^{1}\left(G, A ; \tau_{s, t}\right) \text {. }
$$

We then apply $p^{-1}$ and conclude that $f_{n} * f \rightarrow m * f$ (multiplication in $\mathscr{l}_{L}(G, A ; \tau)$ ) for all $f$ in $L^{1}(V(s, t), A)$. As in Lemma 3 of [2], we can show that finite linear combinations of functions in $L^{1}(V(s, t)$, as $t$ varies over $M(s)$, are dense in $L^{1}(G, A)$ and so $f_{n} \rightarrow m$ strongly in $L^{1}(G, A ; \tau)$. Finally, by using (**) and applying $p^{-1}$, we conclude that $\|m\|=\sup _{\|\mid f\| \leq 1}\{\|m * f\|\}$ where $f$ belongs to $L^{1}(G, A ; \tau)$. This completes the lemma.

At this point it might seem that we should take an arbitrary strongly Cauchy sequence in $L^{1}(G, A ; \tau)$ and successively restrict to sets $U(s)$ and use the above lemma. Unfortunately, the restriction of a strongly Cauchy sequence to a subset (even relatively compact open subset) need not be Cauchy. We must therefore proceed by means of continuous partitions of unity and the following lemma.

Lemma 4.9. Let $\left(f_{n}\right)_{1 \leqq n<\infty}$ be a strongly Cauchy sequence in $L^{1}(G, A ; \tau)$ with $\left\|f_{n}\right\|_{1} \leqq 1$ for all $n$. Then there is a subsequence $\left(\bar{f}_{k}\right)_{1 \leqq k<\infty}$ such that for all $f \in K(G),\left(f \cdot \bar{f}_{k}\right)_{1 \leqq k<\infty}$ is strongly Cauchy, (where $\left.\left(f \cdot \bar{f}_{k}\right)(x)=f(x) \bar{f}_{k}(x)\right)$.

Proof. Let $\left(g_{s}\right)_{1 \leqq s<\infty}$ be a $\|\cdot\|_{\infty}$ dense sequence in $C_{\infty}(G, A)$ (continuous functions vanishing at infinity). Now Proposition 3.4, $\mathrm{d}$ shows that any norm Cauchy sequence in $L^{1}(G, A ; \tau)$ has a subsequence which converges a.e. Thus there is a set $T(1)$ of Haar measure zero and a subsequence $f(n, 1)_{1 \leqq n<\infty}$ of $\left(f_{n}\right)$ such that $\left(f(n, 1) * g_{1}\right)(x)$ converges in $A$ when $x \notin T(1)$. By induction there are sets $T(s)$ of measure zero and subsequences $(f(n, s))_{1 \leqq n<\infty}$ of $\left(f_{n}\right)$ such that (i) $(f(n, s))_{1 \leqq n<\infty}$ is a subsequence of $(f(n, k))_{1 \leqq n<\infty}$ whenever $k \leqq s$ and

(ii) $\left(f(n, s) * g_{s}\right)(x)$ converges when $x \in T(s)$.

It follows in the usual way that if $\bar{f}_{n}=f(n, n)$ and $T=\bigcup_{s=1}^{\infty} T(s)$, 
then $\left(\bar{f}_{n} * g_{t}\right)(x)$ converges (in $\left.n\right) \forall s$ and $\forall x \notin T$. Also, since $\left(g_{s}\right)_{1 \leqq s<\infty}$ is $\|\cdot\|_{\infty}$ dense in $K(G, A)$, it follows easily that if $x \notin T$, then $\left(\bar{f}_{n} * g\right)(x)$ converges for all $g$ in $K(G, A)$. Now if $f \in K(G), g \in K(G, A), x \notin T$, then the function $g^{x}$ defined by: $g^{x}(y)=f\left(x y^{-1}\right) g(y)$ is in $K(G, A)$ and so $\left(\bar{f}_{n} * g^{x}\right)(x)$ converges.

We have

$$
\begin{aligned}
\left(\bar{f}_{n} * g^{x}\right)(x) & =\int_{G} \bar{f}_{n}(y)\left[T(y) g^{x}\left(y^{-1} x\right)\right] \alpha\left(y, y^{-1} x\right) d y \\
& =\int_{G} f(y) \bar{f}_{n}(y)\left[T(y) g\left(y^{-1} x\right)\right] \alpha\left(y, y^{-1} x\right) d y=\left(\left(f \cdot \bar{f}_{n}\right)^{*} g\right)(x)
\end{aligned}
$$

Since $T$ has measure zero, $\left(\left(f \cdot \bar{f}_{n}\right) * g\right)$ converges a.e., and since these functions are all bounded by $\|f\|_{\infty}\|g\|_{\infty}$ and have support contained in $\operatorname{supp}(f) \cdot \operatorname{supp}(g)$, the Lebesgue theorem implies that $\left(f \cdot \bar{f}_{n} * g\right)$ converges in $L^{1}(G, A ; \tau)$. Since $K(G, A)$ is dense in $L^{1}(G, A ; \tau)$, we see that $\left(f \cdot \bar{f}_{i}\right)_{1 \leqq i<\infty}$ is strongly Cauchy $\forall f \in K(G)$.

We now come to the main result.

THEOREM 4.10. $\mathscr{M}_{L}(G, A ; \tau)$ is isometrically isomorphic with

$$
\mathscr{C}_{L}\left(L^{1}(G, A ; \tau)\right) \forall \tau \in Z^{2}(G, A) \text {. }
$$

Proof. We will verify that the hypotheses of Proposition 1.5 hold.

Since $G$ is second countable and paracompact, we can reduce the covering $(W(s))_{s \in G}$ to a locally-finite, countable covering $\left(W\left(s_{n}\right)\right)_{1 \leq n<\infty}$. We can then find a continuous partition of unity $\sum_{n} F_{n}$ subordinate to this covering. Now let $\left(f_{n}\right)_{1 \leqq n<\infty}$ be any strongly Cauchy sequence in $L^{1}(G, A ; \tau)$ with $\left\|f_{n}\right\|_{1} \leqq 1 \forall n$. By Lemma 4.9 there is a subsequence $\left(\bar{f}_{n}\right)_{1 \leqq n<\infty}$ such that $\left(F_{k} \cdot \bar{f}_{n}\right)_{1 \leqq n<\infty}$ is strongly Cauchy for all $k$. Now $\operatorname{supp}\left(F_{k} \cdot \bar{f}_{n}\right) \subset W\left(s_{k}\right)$ for all $k$, and so by Lemma 4.8 , there is a sequence $\left(m_{k}\right)_{1 \leqq k<\infty}$ in $\mathscr{C}_{L}(G, A ; \tau)$ such that $\operatorname{supp}\left(m_{k}\right) \subset W\left(s_{k}\right)$ and $\left(F_{k} \cdot \bar{f}_{n}\right)$ converges strongly to $m_{k}$ as $n \rightarrow \infty$. The fact that $\sum_{k=1}^{\infty} F_{k} \cdot \bar{f}_{n}=\bar{f}_{n}$ for all $n$ implies that $\sum_{k=1}^{\infty} m_{k}(S)$ converges $\forall S \in B(G)$, and the resulting measure $m(m(S)=$ $\left.\sum_{k=1}^{\infty} m_{k}(S)\right)$ belongs to $\mathscr{A}_{L}(G, A ; \tau)$. Finally if $g \in L^{1}(G, A ; \tau), m * g=$ $\sum_{k=1}^{\infty} m_{k} * g=\sum_{k=1}^{\infty} \lim _{n \rightarrow \infty}\left(F_{k} \cdot \bar{f}_{n}\right) * g=\lim _{n \rightarrow \infty} \sum_{k=1}^{\infty}\left(F_{k} \cdot \bar{f}_{n}\right) * g=\lim _{n \rightarrow \infty} \bar{f}_{n} * g$ and so $\left(\bar{f}_{n}\right)$ converges strongly to $m$. Since $\left(f_{n}\right)_{1 \leqq n<\infty}$ is strongly Cauchy, $m=$ strong- $\lim _{n \rightarrow \infty} f_{n}$. Also $\left({ }^{* *}\right)$ implies that $\|m\|=$ $\sup _{\|f\| \leq 1}\|m * f\|, f \in L^{1}(G, A ; \tau)$, and any $m$ can be obtained this way, thus Proposition 1.5 gives the result.

We now investigate the isometric left centralizers on $L^{1}(G, A ; \tau)$ (recall that $m$ is isometric if $\|m * f\|=\|m\|\|f\|=\|f\| \forall f \in L^{1}(G, A ; \tau)$ ).

Lemma 4.11. Suppose that $m \in \mathscr{C}_{L}(G, A ; \tau)$ is an isometric left 
centralizer, and $S \in B(G)$. Then if $n=m \mid S$ (the restriction of $m$ to $S$, i.e., $(m \mid S)(T) \equiv m(T \cap S))$ is not zero, $n /\|n\|$ is isometric.

Proof. Let $T$ be the complement of $S$ in $G$, and let $p=m \mid T$. If $p=0$, then $n=m$ and we are done. If not, then

$$
\begin{aligned}
\|f\| & =\|m * f\| \leqq\|n * f\|+\|p * f\| \leqq\|n\|\|f\|+\|p\|\|f\| \\
& =(\|n\|+\|p\|)\|f\|=\|m\|\|f\|=\|f\| .
\end{aligned}
$$

Thus

$$
\left\|\frac{n}{\|n\|} * f\right\|=\|f\|
$$

and

$$
\left\|\frac{p}{\|p\|} * f\right\|=\|f\|
$$

for all $f \in L^{1}(G, A ; \tau)$.

Lemma 4.12. If $x \in G$, let $\delta_{x} \in \mathscr{l}_{L}(G, A ; \tau)$ be given by: $\delta_{x}(h)=$ $h(x), h \in K(G, A)$.

Then

(a) $\delta_{x}$ is isometric and

(b) if $m$ is isometric $\left(m \in \mathscr{K}_{L}(G, A ; \tau)\right)$ then $\delta_{x} * m$ is isometric.

Proof. (a) $\left(\delta_{x}^{*} f\right)(z)$ is easily computed to be $T(x)\left(f\left(x^{-1} z\right)\right) \alpha\left(x, x^{-1} z\right)$ for all $f \in K(G, A)$. Then

$$
\left\|\delta_{x} * f\right\|=\int_{G}\left\|\left(\delta_{x} * f\right)(z)\right\| d z=\int_{G}\left\|f\left(x^{-1} z\right)\right\|=\|f\|
$$

any by denseness this holds for all $f$ in $L^{1}(G, A ; \tau)$.

(b) is trivial.

Lemma 4.13. Let $m \in \mathscr{M}_{L}(G, A ; \tau), x_{0} \in G$.

Then $\operatorname{supp}\left(\delta_{x_{0}} * m\right)=x_{0} \cdot \operatorname{supp}(m)$.

Proof. Suppose $U$ is an open set with $U \cap \operatorname{supp}(m)=\phi, g$ is a bounded Borel function from $G$ to $A$ with support in $x_{0} U$, and $g^{x_{0}}$ is the function such that $g^{x_{0}}(y)=g\left(x_{0} y\right)$. Then supp $\left(g^{x_{0}}\right)=x_{0}^{-1} \operatorname{supp}(g) \subset$ $U$, and since

$$
\operatorname{supp}\left(x_{0} \cdot m\right)=\operatorname{supp}(m),\left(\delta_{x_{0}} * m\right)(g)=\int_{G} \alpha\left(x_{0}, y\right) g^{x_{0}}(y) d\left(x_{0} \cdot m\right)(y)=0 .
$$

This means that $x_{0} U \cap \operatorname{supp}\left(\delta_{x_{0}} * m\right)=\dot{\phi}$ and so $\operatorname{supp}\left(\delta_{x_{0}} * m\right) \subset x_{0} \operatorname{supp}(m)$. 
The reverse inequality is proved similarly.

The first part of the next lemma is a vector generalization of [21], Theorem 3 .

LEMMA 4.14. Let $V$ be an open, relatively compact neighborhood of e such that $V=V^{-1}$ and $\bar{V}^{2} \subset N$. Let $m$ be an isometric measure with support contained in $V$. Then $m$ has a one point support.

Proof. Suppose that $x_{1} \neq x_{2}$ are two points in $V$. Choose neighborhoods $W_{1}$ and $W_{2}$ of $x_{1}$ and $x_{2}$ respectively such that $W_{1} \cap W_{2}=\phi$ and $W_{i} \subset V, i=1,2$. Choose $\gamma \in K(G)$ such that $\operatorname{supp}(\gamma) \subset N$ and $\gamma \equiv$ 1 on $\bar{V}^{2}$, and let $f \in K(G, A)$, such that $\operatorname{supp}(f) \subset V$. Then

$$
\begin{aligned}
& \|f\|=\|m * f\|=\int_{G}\left\|\int_{G}\left(T(y) f\left(y^{-1} x\right)\right) \alpha\left(y, y^{-1} x\right) d m(y)\right\| d y \\
\leqq & \int_{G} \int_{G}\left\|f\left(y^{-1} x\right)\right\| d|m|(y) d x=\int_{G} \int_{G}\left\|f\left(y^{-1} x\right)\right\| d x d|m|(y) \\
= & \|f\||m|(G)=\|f\|\|m\|=\|f\| .
\end{aligned}
$$

Thus

$$
\left\|\int_{G}\left(T(y) f\left(y^{-1} x\right)\right) \alpha\left(y, y^{-1} x\right) d m(y)\right\|=\int_{G}\left\|f\left(y^{-1} x\right)\right\| d|m|(y)
$$

for almost all $x$. These integrals vanish unless $x \in V^{2}$. Also

$$
\begin{aligned}
& \int_{G}\left(T(y)\left(f\left(y^{-1} x\right)\right)\right) \alpha\left(y, y^{-1} x\right) d m(y) \\
= & \int_{G} \gamma(y)\left(T(y) f\left(y^{-1} x\right)\right) \alpha\left(y, y^{-1} x\right) d m(y)
\end{aligned}
$$

which is continuous everywhere. Thus by continuity, the integrals are equal at all points of $V$. In particular at $x=e$,

$$
\left\|\int_{G}\left(T(y) f\left(y^{-1}\right)\right) \alpha\left(y, y^{-1}\right) d m(y)\right\|=\int_{G}\left\|f\left(y^{-1}\right)\right\| d|m|(y) .
$$

If $\bar{f} \in K(G, A)$ with $\operatorname{supp}(\bar{f}) \subset V$, then let

$$
f(y)=T\left(y^{-1}\right)^{-1}\left[\bar{f}\left(y^{-1}\right) \alpha\left(y^{-1}, y\right)^{*}\right]
$$

in the above and we get:

$$
\left\|\int_{G} \bar{f}(y) d m(y)\right\|=\int_{G}\|\bar{f}(y)\| d|m|(y) .
$$

Finally we get that if $f: G \rightarrow A$ is any bounded Borel function such that $\operatorname{supp}(f) \subset V$, 


$$
\left\|\int_{G} f(y) d m(y)\right\|=\left\|\int_{G}\right\| f(y) \| d|m|(y) .
$$

Let $a, b \in A$ and $f=a \chi_{w_{1}}+b \chi_{w_{2}}$. Then

$$
\begin{aligned}
& \left\|a m\left(W_{1}\right)+b m\left(W_{2}\right)\right\|=\left\|\int f(y) d m(y)\right\|=\int\|f(y)\| d|m|(y) \\
= & \|a\||m|\left(W_{1}\right)+\|b\||m|\left(W_{2}\right) \geqq\|a\|\left\|m\left(W_{1}\right)\right\|+\|b\|\left\|m\left(W_{2}\right)\right\| \\
\geqq & \left\|a m\left(W_{1}\right)+b m\left(W_{2}\right)\right\|
\end{aligned}
$$

and so $\forall a, b \in A$,

$$
\left\|a m\left(W_{1}\right)+b m\left(W_{2}\right)\right\|=\|a\|\left\|m\left(W_{1}\right)\right\|+\|b\|\left\|m\left(W_{2}\right)\right\| \cdot
$$

By Proposition 1.7, this implies that $m\left(W_{1}\right)=0$ or $m\left(W_{2}\right)=0$. Since $x_{1}, x_{2}, w_{1}, w_{2}$ are arbitrary, supp $(m)$ consists of at most one point.

Theorem 4.15. The isometric left centralizers on $L^{1}(G, A ; \tau)$ are precisely the measures $\delta_{x_{0}} \otimes u$, with $x_{0} \in G$ and $u$ an isometric element in $\mathscr{C l}_{L}(A)\left(\right.$ if $\left.h \in K(G, A),\left(\delta_{x_{0}} \otimes u\right)(h)=u \cdot h\left(x_{0}\right)\right)$.

Proof. Suppose that $x$ and $y \neq x$ are in $\operatorname{supp}(m)$, where $m \in$ $\mathscr{C}_{L}(G, A ; \tau)$ is isometric. Choose a neighborhood $V$ of $e$ such that;

(i) $V=V^{-1} ; \bar{V}^{2} \subset N$ and

(ii) $x V \cap y V=\phi$

and let $n, p, q$ be respectively the normalized restrictions of $m$ to $x V, y V$, and $x V \cup y V$. Clearly $p+q=n$, and $p, q, n$ are all isometric by Lemma 4.11 .

Lemmas 4.12, 4.13 and 4.14 imply that $\delta_{x-1} * p$ and $\delta_{y-1} * q$ have one point supports; thus $p$ and $q$ have one point supports, and so $n=c \otimes \delta_{s}+$ $d \otimes \delta_{t}$ for some $s, t \in G$. By translation we may assme $s=e$, and by Corollary $1.8\left\|n m^{\prime}\right\|=\|n\|\left\|m^{\prime}\right\|=\left\|m^{\prime}\right\|$ for all $m^{\prime} \in \mathbb{A}_{L}(G, A ; \tau)$. We choose any $a, b \in A$, and let $m^{\prime}=a \otimes \delta_{e}+b \otimes \delta_{t^{-1}}$. Then

$$
\begin{aligned}
& \|a\|+\|b\|=\left\|m^{\prime}\right\|=\left\|n m^{\prime}\right\| \\
& =\left\|\left[c a+d T(t)(b) \alpha\left(t, t^{-1}\right)\right] \otimes \delta_{e}+c b \otimes \delta_{t^{-1}}+d a \otimes \delta_{t}\right\| \\
& \leqq\left\|c a+d T(t)(b) \alpha\left(t, t^{-1}\right)\right\|\|+\| c\|\| b\|+\| d\|\| a \| \\
& \leqq\|c\|\|a\|+\|d\|\left\|T(t)(b) \alpha\left(t, t^{-1}\right)\right\|+\|c\|\|b\|+\|d\|\|a\| \\
& =(\|c\|+\|d\|)(\|a\|+\|b\|)=\|a\|+\|b\| .
\end{aligned}
$$

In particular, since $b^{\prime}=T(t)(b) \alpha\left(t, t^{-1}\right)$ ranges over all of $A$ as $b$ does, we have $\left\|c a+a b^{\prime}\right\|=\|c\|\|a\|+\|d\|\left\|b^{\prime}\right\|$ for all $a$ and $b^{\prime}$ in $A$. By Proposition $1.9, c=0$ or $b=0$, i.e., $p=0$ or $q=0$ which is a contradiction. Thus $m$ has a one point support and equals $u \otimes \delta_{x}$ for some $x \in G$ and $u \in \mathscr{C}_{L}(A)$. Let $a \in A$ and $n=T(x)^{-1} a \otimes \delta_{e}$. Then $\|a\|=\|n\|=\|m n\|=\|u a\|$ and so $u$ is isometric and we are done. 
We now consider the double centralizers of $L^{1}(G, A ; \tau)$. We know by Proposition 1.2, (iv), that $\mathscr{C}\left(L^{1}(G, A ; \tau)\right)$ may be thought of as the set of all $m \in \mathscr{A}_{L}(G, A ; \tau)$ such that $f^{*} m \in L^{1}(G, A ; \tau)$ for all $f$ in that algebra. The most natural candidate for this algebra is the set $\mathscr{C}(G, A ; \tau)$ of measures in $\mathscr{L}_{L}(G, A ; \tau)$ with values in $\mathscr{C}(A) \subseteq$ $\mathscr{M}_{L}(A)$. We cannot prove, in general, that $L^{1}(G, A ; \tau)$ is a two-sided ideal in $\mathscr{C}(G, A ; \tau)$ or that if $\mathscr{M}(A)=\mathscr{C}_{L}(A)$, then $\mathscr{C}_{L}(G, A ; \tau)$ is the double centralizer algebra of $L^{1}(G, A ; \tau)$ (although this is true if $A=C)$. The presence of the pair $\tau$ causes serious problems which we do not know how to overcome. We give some partial results.

Definition 4.16. We say that a measure $m \in \mathscr{L}_{L}(G, A ; \tau)$ is decomposable if there is a $\nu \in \mathscr{M}(G)$ (the regular, complex, Borel measures of bounded variation on $G$ and a Borel function $g: G \rightarrow$ $\mathscr{C}_{L}(A)$ such that for all $g \in K(G, A), m(h)=\int_{G} g(x) h(x) d \nu(x)$.

Proposition 4.17. If $m$ is decomposable and has values in $\mathscr{L}(A)$, then $f * m$ is in $L^{1}(G, A ; \tau)$ for all $f$ in $L^{1}(G, A ; \tau)$.

Proof. Suppose $m$ is decomposed by $(g, \nu)$ as above, and $m$ (so also $\nu$ ) has compact support. Let $f \in K(G, A)$ and $c=\max \{\Delta(x) \mid x \in$ $\operatorname{supp}(m)\}(\Delta \equiv$ the modular function on $G)$. Finally let $k: G \rightarrow A$ be defined by:

$$
k(y)=\int_{G} \Delta(x) f\left(y x^{-1}\right)\left(T\left(y x^{-1}\right) g(x)\right) \alpha\left(y x^{-1}, x\right) d \nu(x) .
$$

Now $k$ vanishes outside $\operatorname{supp}(f) \cdot \operatorname{supp}(m)$, and on this set $\|k(y)\| \leqq$ $c\|f\|_{\infty}\|m\|<+\infty$, so $k \in L^{1}(G, A ; \tau)$. Now let $h \in K(G, A)$. Then

$$
\begin{aligned}
(f * m)(h) & =\int_{G} \int_{G} f(y)(T(y) g(x)) \alpha(y, x) h(y x) d \nu(x) d y \\
& =\int_{G} \int_{G} f(y)(T(y) g(x)) \alpha(y, x) h(y x) d y d \nu(x) \\
& =\int_{G} \int_{G} \Delta(x) f\left(y x^{-1}\right)\left(T\left(y x^{-1}\right) g(x), \alpha\left(y x^{-1}, x\right) d \nu(x) h(y) d y\right. \\
& =\int k(y) h(y) d y=k(h) .
\end{aligned}
$$

Then by Lemma $4.6 f * m=k \in L^{1}(G, A ; \tau)$.

Since $K(G, A)$ is norm dense in $L^{1}(G, A ; \tau)$, and measures with compact support are norm dense in $\mathscr{L}(G, A ; \tau)$, we are done.

Corollary 4.18. It $\mathscr{t}(A)=\mathscr{M}_{L}(A)$, and every measure in $\mathscr{A}_{L}(G, A ; \tau)$ is decomposable, then $\mathscr{M}\left(L^{1}(G, A ; \tau)\right)=\mathscr{C}(G, A: \tau)$. 
The decomposable measures form a large class, and Proposition 4.17 is useful, but we do not know conditions which will guarantee that every measure is decomposable.

Proposition 4.19. The unitary double centralizers on $L^{1}(G, A ; \tau)$ are precisely the measures $u \otimes \delta_{x}$ where $u \in \mathscr{U}(A)$.

Proof. Unitaries are isometric, so every unitary has the form $u \otimes \delta_{x}$ for an isometric $u$. Since $\left(u \otimes \delta_{x}\right)$ is invertible, it is not hard to see that $u^{-1}$ exists. Now $\left(u \otimes \delta_{x}\right)^{*}$ is uniquely defined by the equation $\left(u \otimes \delta_{x}\right) * f=\left(f^{*} * u \otimes \delta_{x}\right) *$ for $f \in L^{1}(G, A ; \tau)$ and a straightforward computation shows that since $\left(u \otimes \delta_{x}\right)^{*}=\left(u \otimes \delta_{x}\right)^{-1}, u=u^{-1}=$ $u^{*} \in \mathscr{Q}_{t}(A)$. Conversely if we are given $u \otimes \delta_{x}$ with $u \in \mathscr{Q}_{L}(A)$, we can compute $\left(u \otimes \delta_{x}\right)^{-1}$ to be $\left(\alpha\left(x^{-1}, x\right)^{*} T\left(x^{-1}\right)\left(u^{*}\right) \otimes \delta_{x^{-1}}\right)$ and a direct computation shows that $f^{*} *\left(u \otimes \delta_{x}\right)=\left[\left(u \otimes \delta_{x}\right)^{-1} * f\right]^{*} \forall f \in L^{1}(G, A ; \tau)$, so $u \otimes \delta_{x}$ is unitary.

\section{Examples and applications.}

EXAMPLE 1. Let $G$ be a locally compact group, $N$ a closed, normal subgroup and $K$ the quotient group $G / N$. Suppose that there is a locally continuous cross section from $K$ to $G$, i.e., $G$ is a "fibred extension". Then it is known (see [4]) that $\exists \tau \equiv(T, \alpha) \in Z^{2}\left(K, L^{1}(N)\right)$ so that $L^{1}(G)$ is isomorphic with $L^{1}\left(K, L^{1}(N) ; \tau\right)$. Since $\mathscr{C}(G)$ is known to be isomorphic with $\mathscr{L}_{L}\left(L^{1}(G)\right)([21]$, Th. 2), our main result implies that $\mathscr{C}(G)$ and $\mathscr{C}_{L}\left(K, L^{1}(N) ; \tau\right)$ are isometrically * isomorphic. This means that each measure on $G$ can be decomposed into a measure on $K$ with values which are measures on $N$, in such a way that norm, sums, and products (convolutions) are preserved. We note that if $N$ above is contained in the center of $G$, then $T$ (in the pair $(T, \alpha)=\tau)$ is trivial and our results make contact with those of Edwards [10]. The precise relation between our results and his, however, is not entirely clear, since the formulations are very different. Moreover, he does not require the existence of a locally continuous cross section, while for groups which have such a cross section, our results are somewhat more explicit.

We consider applications of our results to isomorphisms of twisted group algebras. Wendel has shown that if $G$ and $G^{\prime}$ are locally compact groups such that $L^{1}(G)$ is isometrically isomorphic with $L^{1}\left(G^{\prime}\right)$, then $G$ is topologically isomorphic with $G^{\prime}$. This would suggest that if $L^{1}(G, A ; \tau)$ and $L^{1}\left(G^{\prime}, A^{\prime} ; \tau^{\prime}\right)$ are isometrically isomorphic then $A$ and $A^{\prime}$ are isometrically isomorphic and $G$ and $G^{\prime}$ are topologically isomorphic. This result, however, is false. In fact let $G$ be a locally compact group which has two normal subgroups $N_{1}, N_{2}$ with $G / N_{1}$ 
and $G / N_{2}$ non-isomorphic. By example one above, $L^{1}\left(G / N_{1}, L^{1}\left(N_{1}\right) ; \tau_{1}\right) \cong$ $L^{1}\left(G / N_{2}, L^{1}\left(N_{2}\right) ; \tau_{2}\right)$ but $G / N_{1} \neq G / N_{2}$. We can, however, classify certain isomorphisms.

DEFINITION 5.1. Let $\sigma$ be an isomorphism from $L^{1}\left(G_{1}, A_{1} ; \tau_{1}\right)$ onto $L^{1}\left(G_{2}, A_{2} ; \tau_{2}\right)$. We say $\sigma$ is special if the canonical lifting of $\sigma$ to the left centralizer algebra takes $A_{1}$ onto $A_{2}$, and similarly for $\sigma^{-1}$ (note that $A$ is contained in $\mathscr{A}_{L}\left(L^{1}(A, G ; \tau)\right)$ through the map $\left.a \rightarrow a \otimes \delta_{e}\right)$. From now on for convenience we will denote by $\bar{\alpha}$ the canonical lifting of an isomorphism $\alpha$ from an algebra to the corresponding left centralizer algebra.

Suppose that $L^{1}\left(G_{i}, A_{i} ; \tau_{i}\right)(i=1,2)$ are two locally continuous twisted group algebras (i.e. $\left.\tau_{i} \in Z^{2}\left(G_{i}, A_{i}\right), i=1,2\right)$. Then (see $\S 2$ ) $\tau_{i}$ extends to a member $\bar{\tau}_{i}$ of $Z^{2}\left(G_{i}, \mathscr{U}\left(A_{i}\right)\right)$, and Proposition 4.19 and the definition of multiplication of measures show that if $E_{\bar{\tau}_{i}}: 0 \rightarrow \mathscr{Q}\left(A_{i}\right) \rightarrow$ $B_{\bar{\tau}_{i}} \rightarrow G_{i} \rightarrow 0$ is the corresponding group extension (see $\S 2$ ), then $B_{\bar{\tau}_{i}}$ is isomorphic with the group of unitary double centralizers on $L^{1}\left(G_{i}, A_{i} ; \tau_{i}\right)$.

Now if $\sigma$ is a special isomorphism from $L^{1}\left(G_{1}, A_{1} ; \tau_{1}\right)$ onto $L^{1}\left(G_{2}, A_{2} ; \tau_{2}\right)$, then $\sigma$ takes $A_{1}$ onto $A_{2}$ and thus $\mathscr{Q}\left(A_{1}\right)$ onto $\mathscr{C}\left(A_{2}\right)$. By Proposition 1.6 (ii) $\sigma$ also takes $B_{\bar{\tau}_{1}}$ isomorphically onto $B_{\bar{\tau}_{2}}$. Thus there is a commutative diagram:

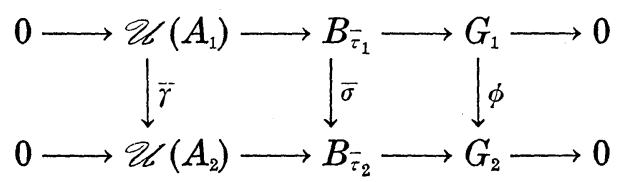

where $\bar{\gamma}$ is the restriction of $\bar{\sigma}$ to $\mathscr{C}\left(A_{1}\right)$ and $\phi$ is the induced map (necessarily bicontinuous) from $G_{1}$ to $G_{2}$.

THEOREM 5.2. (a) $L^{1}\left(G_{1}, A_{1} ; \tau_{1}\right)$ and $L^{1}\left(G_{2}, A_{2} ; \tau_{2}\right)$ are specially isomorphic if and only if there are isomorphisms $\phi: G_{1} \rightarrow G_{2}$ and $\gamma$ : $A_{1} \rightarrow A_{2}$ such that if $s=(\phi, \gamma),\left[\tau_{1}\right]=\left[\tau_{2}\right]^{s}$.

(b) If the above holds and $s=(\phi, \gamma)$ is as before, let $p \in F\left(G_{1}, A_{1}\right)$ be such that $p \cdot\left(s \cdot \tau_{2}\right)=\tau_{1}$, and let $c \in R^{+}$be defined by the condition that $\mu_{1}(S)=c \mu_{2}(\phi(S))\left(S \in B\left(G_{1}\right), \mu_{i}\right.$ Haar measure in $\left.G_{i}\right)$. Then $\sigma^{p}(f)(\phi(x))=$ $c \gamma(f(x) p(x))$ defines a map $\sigma_{s}^{p}: L^{1}\left(G_{1}, A_{1} ; \tau_{1}\right) \rightarrow L^{1}\left(G_{2}, A_{2} ; \tau_{2}\right)$ which is a special isomorphism, and all such isomorphisms arise in this way.

Proof. The discussion immediately preceding this theorem shows that if a special isomorphism $\sigma: L^{1}\left(G_{1}, A_{1} ; \tau_{1}\right) \rightarrow L^{1}\left(G_{2}, A_{2} ; \tau_{2}\right)$ exists, it induces an isomorphism $(\bar{\gamma}, \bar{\sigma}, \phi)$ between $E_{\bar{\tau}_{1}}$ and $E_{\bar{\tau}_{2}}$. Proposition 2.7 then shows that $\left[\bar{\tau}_{1}\right]=\left[\bar{\tau}_{2}\right]^{s}$, where $\bar{s}=(\phi, \bar{\gamma})$. Since $\bar{\gamma}$ can be considered to be the extension to $\mathscr{C}\left(A_{1}\right)$ of an isomorphism $\gamma: A_{1} \rightarrow A_{2}$ (also induced by $\bar{\sigma}$ ) it follows that $\left[\tau_{1}\right]=\left[\tau_{2}\right]^{s}$, where $s=(\phi, \gamma)$. 
Now if there is a pair $s=(\phi, \gamma)$ as above, with $\left[\tau_{1}\right]=\left[\tau_{2}\right]^{s}$, and if $p \in F\left(G_{1}, A_{1}\right)$ gives the equivalence between $\tau_{1}$ and $\tau_{2}^{s}$, then for $f$, $g$ in $L^{1}\left(G_{1}, A_{1} ; \tau_{1}\right):\left[\sigma_{s}^{p}(f) * \sigma_{s}^{p}(g)\right](\phi(x))$

$$
\begin{aligned}
& =c^{2} \gamma\left[\int_{G_{1}}(f(y) p(y)) \gamma^{-1}\left(T_{2}(\phi(y)) \gamma\left(g\left(y^{-1} x\right) p\left(y^{-1} x\right)\right) \alpha_{2}\left(\dot{\phi}(y), \dot{\phi}\left(y^{-1} x\right)\right) d \gamma(y)\right]\right. \\
& =c \gamma\left[\int_{G_{1}}(f \cdot p)(y) T_{2}^{s}(y)\left[(g \cdot p)\left(y^{-1} x\right)\right] \alpha_{2}^{s}\left(y, y^{-1} x\right) d y\right] \\
& =c \gamma\left[\int_{G_{1}}(f \cdot p)(y) T_{2}^{s}(y)\left(g\left(y^{-1} x\right)\right) p(y)^{*}\left[p(y) T_{2}^{s}(y)\left(p\left(y^{-1} x\right)\right)\right]\right. \\
& \left.\quad \times \alpha_{2}^{s}\left(y, y^{-1} x\right) p(x)^{*} p(x) d y\right] \\
& =c \gamma\left[\int_{G_{1}} f(y) T_{1}(y)\left[g\left(y^{-1} x\right)\right] \alpha_{1}\left(y, y^{-1} x\right) d y p(x)\right] \\
& =c \gamma\left(\left(f_{1} * g\right)(x) p(x)\right)=\sigma_{s}^{p}\left(f_{1} * g\right)(\phi(x)) .
\end{aligned}
$$

Also

$$
\begin{aligned}
& \left\|\sigma_{s}^{p}(f)\right\|_{1}=c \int_{G_{2}}\|\gamma(f(x) p(x))\| d \phi(x) \\
= & \left.\int_{G_{1}}\|f(x)\| d x=\|f\|_{1} \text { (since } d \phi(x)=c^{-1} d x\right) .
\end{aligned}
$$

Finally if $g \in L^{1}\left(G_{2}, A_{2} ; \tau_{2}\right)$ and $f$ is defined by: $f(x)=c^{-1} \gamma^{-1}[g(\phi(x))] p(x)^{-1}$, then $f \in L^{1}\left(G_{1}, A_{1} ; \tau_{1}\right)$ and $\sigma_{s}^{p}(f)=g$. Hence $\sigma_{s}^{p}$ is an (obviously special) isomorphism. Now it is trivial to show that $\sigma_{s}^{p}$ produces (by the process outlined at the start of this proof) the pair s. We will thus be finished if we show that for any special isomorphism $\sigma$, producing a pair $s=(\phi, \gamma), \sigma \equiv \sigma_{s}^{p}$, for some $p$. Direct computation shows that if we let $p$ be defined by the equation, $\bar{\sigma}\left(\delta_{x}\right)=p(x) \delta_{\phi(x)}$, then $\bar{\sigma}$ and $\bar{\sigma}_{s}^{p}$ will agree on measures in $\mathscr{C}\left(G_{1}, A_{1} ; \tau_{1}\right)$ with finite support. It is easily seen that these are strongly dense, hence $\sigma=\sigma_{s}^{p}$, and we are done.

COROLlary 5.3. The set of special isomorphism classes of twisted group algebras over $(G, A)$ is in one-to-one correspondence with the set of orbits in $H^{2}(G, A)$ under the action of $\mathscr{G}(G, A)(\equiv \mathscr{A}(G) \times$ $\mathscr{A}(A))$.

These results generalize results of Wendel [21], and Edwards and Lewis [11], as previously mentiond. It is worth remarking that our method of proof, which involves going to associated group extensions, gives an almost immediate proof of the continuity properties of $\phi, \gamma$, $p$ associated with $\sigma$. This was one of the most difficult parts of the proofs of the above mentioned special cases, and our approach seems more natural as well as easier, even in those special cases.

We can also describe the group of special automorphisms of a fixed algebra $L^{1}(G, A ; \tau)$. 
Proposition 5.4. Suppose $L^{1}(G, A ; \tau)$ is given and $\mathscr{G}_{\tau}$ represents

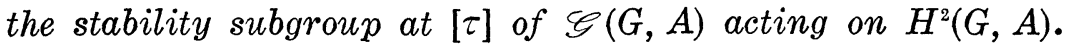

Let $\mathscr{A}(G, A ; \tau)$ be the group of special automorphisms of $L^{1}(G, A ; \tau)$. Then there is an exact sequence $0 \rightarrow Z^{1}(G, C(A)) \rightarrow \mathscr{A}(G, A ; \tau) \rightarrow \mathscr{S}: \rightarrow 0$, i.e., $\mathscr{A}(G, A ; \tau)$ is an extension of $\mathscr{C}_{\tau}$ by $Z^{1}(G, C(A))$.

Proof. If $\sigma_{1}, \sigma_{2} \in \mathscr{A}(G, A ; \tau)$, then by Theorem 5.2, there are pairs $s_{i}=\left(\phi_{i}, \gamma_{i}\right) \in \mathscr{G}_{\tau}$ and $p_{i}: G \rightarrow \mathscr{U}(A)(i=1,2)$, so that $\sigma_{i}=\sigma_{s_{i}}^{p_{i}}$.

It is not hard to show that $\left(\sigma_{2} \sigma_{1}\right)(f)(z)$

$$
=c_{2} c_{1} \gamma_{2} \gamma_{1}\left[f\left(\left(\phi_{2} \phi_{1}\right)^{-1}(z)\right) p_{3}\left(\left(\phi_{2} \phi_{1}\right)^{-1}(z)\right)\right]
$$

(where $p_{3}(x)=p_{1}(x)\left[\gamma_{1}^{-1} p_{2}\left(\phi_{1}(x)\right)\right]$ ), and so $\sigma_{2} \sigma_{1}$ corresponds to the pair $\left(\phi_{1} \phi_{2}, \gamma_{1} \gamma_{2}\right)$. Thus the natural map from $\mathscr{A}(G, A ; \tau)$ to $\mathscr{G}_{\tau}$ is an onto homomorphism. If $\sigma_{e}^{p}$ is in the kernel of this map, then $p \cdot \tau=\tau$ and so (see remark following Lemma 2.3) $p \in Z^{1}(G, C(A)$ ).

If $A$ is an abelian Banach algebra, the above results simplify somewhat.

ExAMPLE 2. Suppose $A$ is abelian. The following results follow from Corollary 5.3, Proposition 5.4. or previous remarks.

(a) If $L^{1}\left(G, A ; T_{1}, \alpha_{1}\right)$ is specially isomorphic with $L^{1}\left(G, A ; T_{2}, \alpha_{2}\right)$ then $T_{1}=T_{2}$.

(b) The special isomorphism classes over $(G, A ; T)$ are in one-toone correspondence with the orbits of $H_{T}^{2}(G, A)$ under $\mathscr{G}(G, A) ;\left[\alpha_{1}\right]$ and $\left[\alpha_{2}\right]$ are in the same orbit if and only if $\exists(\phi, \gamma) \in \mathscr{G}(G, A)$ such that $\alpha_{2} \circ \phi$ and $\gamma \circ \alpha_{1}$ are cohomologous.

(c) If $L^{1}(G, A ; \tau)$ is fixed with $A$ abelian and $\tau=(T, \alpha)$ then the special automorphisms of $L^{1}(G, A ; \tau)$ are given by triples $(\phi, \gamma, \zeta)$ where

(i ) $(\phi, \gamma) \in \mathscr{G}(G, A)$ and $T(\phi(x)) \alpha(a)=\gamma(T(x) a)$

(ii) $\zeta: G \rightarrow \mathscr{U}(A)$

(iii) if $\beta(x, y)$ is the cocycle $\alpha(\phi(x), \phi(y)) \gamma\left(\alpha^{-1}(x, y)\right)$ then $\beta=\partial \zeta$, the coboundary of $\zeta$.

Up to now we have dealt only with special isomorphisms. Some of the most interesting examples of twisted group algebras occur when the object algebra is a $C^{*}$-algebra (covariance algebras, transformation group algebras, etc.). We show that for certain $C^{*}$-algebras, all isomorphisms are special, and so our previous results hold in complete generality.

THEOREM 5.5. Let $A_{1}$ and $A_{2}$ be $C^{*}$-algebras. Let $\sigma$ be an isomorphism of $L^{1}\left(G_{1}, A_{1} ; \tau_{1}\right)$ with $L^{1}\left(G_{2}, A_{2} ; \tau_{2}\right)\left(\tau_{i} \in Z^{2}\left(G_{i}, A_{i}\right), i=1,2\right)$.

Then $\sigma\left(\mathscr{L}\left(A_{1}\right)\right)=\mathscr{M}\left(A_{2}\right)$. 
Proof. By [1], 2.11, $\mathscr{C}\left(A_{1}\right)$ is a $C^{*}$-algebra with identity and so by [9], $1, \S 1$, Proposition 3 , every element in $\mathscr{M}\left(A_{1}\right)$ is a complex linear combination of at most four unitaries in $\mathscr{Q}_{6}\left(A_{1}\right)$. It is therefore sufficient to prove that $\sigma\left(\mathscr{U}\left(A_{1}\right)\right) \subset \mathscr{C}\left(A_{2}\right)$. Suppose $u \in \mathscr{U}\left(A_{1}\right)$ and $v \equiv$ $\sigma(u)=a \otimes \delta_{x}$, where $a \in \mathscr{U}\left(A_{2}\right), x \in G_{2}, x \neq e$ (the identity in $G_{2}$ ). We know that $\sigma(u)$ is unitary and so by Proposition 4.19 it must be in the above form for some $x$. Choose complex numbers $\beta, \gamma, \rho$ of modulus one such that: (a) $\bar{\beta} \gamma+\bar{\gamma} \rho=0$, and (b) $a(T(x) \alpha) \alpha(x, x) \neq(-\beta / \rho) I$, where $I$ is the identity in $\mathscr{C}\left(A_{2}\right)$. Condition $(b)$ is possible whether or not $a(T(x) a) \alpha(x, x)$ is a multiple of $I$. Let $z=\beta+\gamma v+v^{2}$. Then (since $v$ is unitary) $z^{*}=\bar{\beta}+\bar{\gamma} v^{-1}+\bar{\rho} v^{-2}$, and $z^{*} z=|\beta|^{2}+|\gamma|^{2}+|\rho|^{2}+$ $(\bar{\beta} \gamma+\bar{\gamma} \rho) v+(\bar{\gamma} \beta+\bar{\rho} \gamma) v^{-1}+\bar{\beta} \rho v^{2}+\bar{\rho} \beta v^{-2}=|\beta|^{2}+|\gamma|^{2}+|\rho|^{2}+(\bar{\beta} \rho) v^{2}+$ $(\bar{\rho} \beta) v^{-2}$ (by (a)). Now $\left\|z^{*} z\right\|=\left\|3+(\bar{\beta} \rho) v^{2}+(\bar{\rho} \beta) v^{-2}\right\|=\| 1+(\beta+$ $\left.\rho v^{2}\right)^{*}\left(\beta+\rho v^{2}\right) \|$. On the other hand, $\|z\|=\left\|\beta+\rho v^{2}\right\|+|\gamma|$ (since the support of the measure $v$ is $x \neq e$, and $\|v\|=1$ ). Thus $\|z\|^{2}=$ $1+2\left\|\beta+\rho v^{2}\right\|+\left\|\beta+\rho v^{2}\right\|^{2}$. Now $\beta+\rho v^{2}=\beta+\rho(a(T(x) a) \alpha(x, x)) \otimes \delta_{x_{2}}$ which is clearly nonzero if $x^{2}=e$, and if $x^{2} \neq e$ the element is nonzero by assumption (b). Thus in any case $\|z\|^{2}>1+\left\|\beta+\rho v^{2}\right\|^{2} \geqq$ $\left\|z^{*} z\right\|=\|z\|^{2}$ which is a contradiction. Thus $x=e$ and $v \in \mathscr{M}\left(A_{2}\right)$. This completes the proof.

THeOREm 5.6. Let $A$ be a $C^{*}$-algebra, $G$ as above, and $\tau=(T, \alpha)$ a twisting pair for $(G, A)$.

(a) Suppose $A$ has the additional property that any automorphism of $\mathscr{C l}(A)$ leaves $A$ invariant.

Then any automorphism of $L^{1}(G, A ; \tau)$ is special.

(b) $A$ satisfies the condition of (a) if

(i) $A$ has identity or

(ii) $A$ is commutative or

(iii) $A$ is primitive and Type $I$.

Proof. (a) follows from Theorem 5.5, and

(b)-(i) is trivial.

(b)-(ii). If $A$ is commutative, $A$ can be assumed to be $C_{\infty}(X)$ for a locally compact, $T_{2}$, second-countable (since $A$ is separable) space $X$. It is then known that $\mathscr{M}(A)=C_{b}(X)=C(Y)$, where $C_{b}(X)$ is the algebra of continuous, bounded, complex-valued functions on $X$, and $Y$ is the Stone-Céck compactification of $X$. Then any isomorphism $\sigma$ of $C(Y)$ comes from a homeomorphism $f$ of $Y$. Now it is well known that $Y$ is not first countable at any points of $Y \dashv X$, and in this case, that fact characterizes $Y \dashv X$. Thus $f$ (and $f^{-1}$ ) leave $X$ invariant and so $\sigma$ must leave $A$ invariant. (b)-(iii). We may assume that $A=C(H)$, the algebra of compact operators on a Hilbert space $H$. Then (see [16], Th. 18), $\mathscr{L}(A)=\mathscr{B}(H)$ (the bounded linear 
operators on $H$ ), and any automorphism of $\mathscr{B}(H)$ is an inner automorphism given by a unitary in $\mathscr{B}(H)$. It is well known that such an automorphism will leave the algebra $C(H)$ invariant. This completes the proof.

REMARK. Very likely a minor modification of the proof of $(b)-$ (ii) would work for any $n$-homogeneous $C^{*}$-algebra.

EXAMPLE 3. Our final example of an application of our results concerns the Homogeneous Banach $*$-algebraic Bundles $(H-B$-bundles) of Fell [12]. We refer the reader to [12] for all definitions connected with this example. Let $\mathscr{B}=\langle B, \pi, \circ, *\rangle$ be an $H-B$-bundle over a locally compact group $G$. We assume as usual that $G$ is secondcountable, and also that the algebra $A \equiv B_{e}$ is separable. We recall that, according to Fells classification of such bundles, [12], $\mathscr{B}$ is canonically associated with a group extension $E(\mathscr{B}): 0 \rightarrow \mathscr{C}(A) \stackrel{i}{\longrightarrow}$ $\mathscr{C}(B) \stackrel{\pi}{\longrightarrow} G \rightarrow 0$, where $\mathscr{C}(A)$ is as usual and $\mathscr{C}(B)$ is the group of "unitary multipliers" of the "bundle space" $B$. We will say that $\mathscr{B}$ is locally trivial if $\pi$ has a Borel right inverse which is continuous in a neighborhood of $e$.

Finally if $L^{1}(B)$ is the "cross sectional algebra" of $B$, and $u \in$ $\mathscr{U}(B)$, there is a canonical element $\zeta(u)$ of $\mathscr{U}\left(L^{1}(B)\right)$ given by: $[\zeta(u) f](x)=$ $u\left[f\left(\pi(u)^{-1} x\right)\right](\pi$ is the canonical map of $\mathscr{U}(B)$ onto $G)$.

Proposition 5.7. If $\mathscr{B}$ is a locally trivial $H-B$ bundle, the above map $\zeta$ is an isomorphism of $\mathscr{C}(B)$ onto $\mathscr{U}\left(L^{1}(B)\right)$.

Proof. [3] shows that there is a locally continuous twisted group algebra $L^{1}(G, A ; \tau)$ isomorphic with $L^{1}(B)$ and that the sequence $0 \rightarrow \mathscr{U}(A) \rightarrow$ $U\left(L^{1}(G, A ; \tau)\right) \rightarrow G \rightarrow 0$ is equivalent with $E(\mathscr{B})$. Proposition 2.6, Th. 4.15, and the discussion surrounding these results complete the proof.

We remark in closing that this proposition may well hold for more general $H-B$-bundles and that whenever $\mathscr{U}(B) \cong \mathscr{U}\left(L^{1}(B)\right)$, our results on special isomorphisms can be reworked to apply to the Bundle $\mathscr{B}$. On the other hand, our main Theorem 4.10 does not seem to have a natural statement in the Bundle context.

Acknowledgment. We would like to thank the referee of this paper for many useful suggestions and improvements. In particular the proof of Lemma 4.6 is due to him and replaces a much longer and more awkward one. 


\section{REFERENCES}

1. R. C. Busby, Double centralizers and extensions of $C^{*}$-algebras, Trans. Amer. Math. Soc., 132, no. 1 (1968), 79-99.

2. - On a theorem of Fell, Proc. Amer. Math. Soc., 30 no. 1 (1971), 133-140.

3. - On the equivalence of twisted group algebras and Banach *-algebraic bundles, submitted to Proc. Amer. Math. Soc.

4. R. C. Busby and H. A. Smith, Representations of twisted group algebras, Trans. Amer. Math. Soc., 149 (1970), 503-537.

5. L. Calabi, Sur les extensions des groupes topologiques, Ann. Math. pura Appl. (4), 32 (1951), 295-370.

6. J. Dauns, Multiplier rings and primitive ideals, Trans. Amer. Math. Soc., 145 (1969), 125-158.

7, J. Dauns and K. H. Hofmann, Spectral theory of algebras and adjunction of identity, to appear.

8. N. Dinculeanu, Vector Measures, Pergamon Press, New York, 1967.

9. J. Dixmier, Les algèbres d'operators daus l'espace Hilbertien (Algèbres de von Newmann), Gauthier-Villars, Paris, 1957.

10. C. M. Edwards, The measure algebra of a central group extension, to appear.

11. C. M. Edwards and J. T. Lewis, Twisted group algebras II, Commun. Math. Phys., 13 (1969), 131-141.

12. J. M. G. Fell, An extension of Mackey's method to Banach *-algebraic bundles, Memoirs Amer. Math. Soc., no. 90 (1969).

13. F. Greenleaf, Norm decreasing homomorphisms of group algebras, Pacific J. Math., 15 (1965), 1187-1219.

14. - Characterization of group algebras in terms of their translation operators, Pacific J. Math., 18 (1966), 243-276.

15. G. Hochschild, Cohomology and representations of associative algebras, Duke Math. J., 14 (1947), 921-948.

16. B. E. Johnson, An introduction to the the theory of centralizers, Proc. London Math. Soc., 14 (1964), 299-320.

17. C Centralizers on certain topological algebras, J. London Math. Soc., (1964), 603-614.

18. H. Leptin, Verallgemeinerte $L^{1}$-algebren, Math. Ann., 159 (1965), 51-76.

19. - Verallgemeinerte $L^{1}$-algebren und projektive darstellungen local kompakter gruppen I, Invent. Math., 3 (1967), 257-281.

20. - Verallgemeinerte $L^{1}$-algebren und projektive darstellungen local kompakter gruppen II, Invent. Math., 4 (1967), 68-86.

21. J. G., Wendel, Left centralizers and isomorphisms of group algebras, Pacific J. Math., 2 (1952), 251-261.

22. - On isometric isomorphism of group algebras, Pacific J. Math., 1 (1951), 305-311.

Received March 20, 1972.

DREXEL UNIVERSITY 


\section{PACIFIC JOURNAL OF MATHEMATICS}

\section{EDITORS}

D. Gilbarg AND J. MILGRAM

Stanford University

Stanford, California 94305

\section{R. A. Beaumont}

University of Washington

Seattle, Washington 98105

\section{J. DUGUNDJI*}

Department of Mathematics University of Southern California Los Angeles, California 90007

RICHARD ARENS

University of California Los Angeles, California 90024

\section{ASSOCIATE EDITORS}
E. F. BECKENBACH
B. H. NEUMANN
F. WOLF
K. YoshidA

\section{SUPPORTING INSTITUTIONS}

\author{
UNIVERSITY OF BRITISH COLUMBIA \\ CALIFORNIA INSTITUTE OF TECHNOLOGY \\ UNIVERSITY OF CALIFORNIA \\ MONTANA STATE UNIVERSITY \\ UNIVERSITY OF NEVADA \\ NEW MEXICO STATE UNIVERSITY \\ OREGON STATE UNIVERSITY \\ UNIVERSITY OF OREGON \\ OSAKA UNIVERSITY
}

\author{
UNIVERSITY OF SOUTHERN CALIFORNIA \\ STANFORD UNIVERSITY \\ UNIVERSITY OF TOKYO \\ UNIVERSITY OF UTAH \\ WASHINGTON STATE UNIVERSITY \\ UNIVERSITY OF WASHINGTON \\ AMERICAN MATHEMATICAL SOCIETY \\ NAVAL WEAPONS CENTER
}

The Supporting Institutions listed above contribute to the cost of publication of this Journal, but they are not owners or publishers and have no responsibility for its content or policies.

Mathematical papers intended for publication in the Pacific Journal of Mathematics should be in typed form or offset-reproduced, (not dittoed), double spaced with large margins. Underline Greek letters in red, German in green, and script in blue. The first paragraph or two must be capable of being used separately as a synopsis of the entire paper. Items of the bibliography should not be cited there unless absolutely necessary, in which case they must be identified by author and Journal, rather than by item number. Manuscripts, in duplicate if possible, may be sent to any one of the four editors. Please classify according to the scheme of Math. Rev. Index to Vol. 39. All other communications to the editors should be addressed to the managing editor, Richard Arens, University of California, Los Angeles, California, 90024.

50 reprints are provided free for each article; additional copies may be obtained at cost in multiples of 50 .

The Pacific Journal of Mathematics is issued monthly as of January 1966. Regular subscription rate: $\$ 48.00$ a year (6 Vols., 12 issues). Special rate: $\$ 24.00$ a year to individual members of supporting institutions.

Subscriptions, orders for back numbers, and changes of address should be sent to Pacific Journal of Mathematics, 103 Highland Boulevard, Berkeley, California, 94708.

PUBLISHED BY PACIFIC JOURNAL OF MATHEMATICS, A NON-PROFIT CORPORATION

Printed at Kokusai Bunken Insatsusha (International Academic Printing Co., Ltd.), 270, 3-chome Totsuka-cho, Shinjuku-ku, Tokyo 160, Japan.

* C. DePrima will replace J. Dugundji until August 1974.

Copyright (C) 1973 by

Pacific Journal of Mathematics

All Rights Reserved 


\section{Pacific Journal of Mathematics}

\section{Vol. 47, No. $2 \quad$ February, 1973}

David Parham Bellamy, Composants of Hausdorff indecomposable continua; a mapping approach ........................ 303

Colin Bennett, A Hausdorff-Young theorem for rearrangement-invariant spaces ...........................................

Roger Daniel Bleier and Paul F. Conrad, The lattice of closed ideals and $a^{*}$-extensions of an abelian l-group ...

Ronald Elroy Bruck, Jr., Nonexpansive projections on subsets of Banach

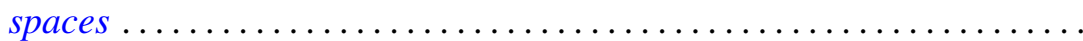

Robert C. Busby, Centralizers of twisted group algebras ............. 357

M. J. Canfell, Dimension theory in zero-set spaces ................ 393

John Dauns, One sided prime ideals ........................ 401

Charles F. Dunkl, Structure hypergroups for measure algebras . . . . . . . . . 413

Ronald Francis Gariepy, Geometric properties of Sobolev mappings ...... 427

Ralph Allen Gellar and Lavon Barry Page, A new look at some familiar spaces of intertwining operators ...........................

Dennis Michael Girard, The behavior of the norm of an automorphism of the

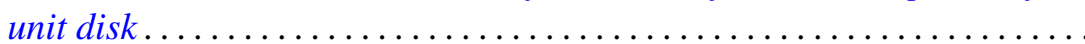

George Rudolph Gordh, Jr., Terminal subcontinua of hereditarily

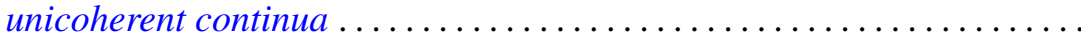

Joe Alston Guthrie, Mapping spaces and cs-networks. .

Neil Hindman, The product of $F$-spaces with $P$-spaces . 473

M. A. Labbé and John Wolfe, Isomorphic classes of the spaces $C_{\sigma}(S)$

Ernest A. Michael, On k-spaces, $k_{R}$-spaces and $k(X) \ldots$

Donald Steven Passman, Primitive group rings .

C. P. L. Rhodes, A note on primary decompositions of a pseudovaluation ...

Muril Lynn Robertson, A class of generalized functional differential equations

Ruth Silverman, Decomposition of plane convex sets. $I$.

Ernest Lester Stitzinger, On saturated formations of solvable Lie algebras................................

B. Andreas Troesch, Sloshing frequencies in a half-space by Kelvin inversion ...

L. E. Ward, Fixed point sets .

Michael John Westwater, Hilbert transforms, and a problem in scattering

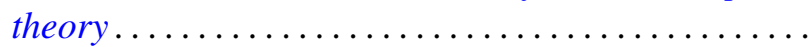

Misha Zafran, On the spectra of multipliers... 